\title{
Innovation expenditures efficiency in Central and Eastern European Countries
}

\author{
Pawel Dobrzanski ${ }^{1}$
}

\begin{abstract}
The purpose of this study is to verify whether the money spend on $R \& D$ are used efficiently in CEE countries. Nowadays, innovativeness is one of the most crucial factors accelerating economic growth. Increasing innovativeness is particularly important for developing countries, where policymakers are implementing various innovation strategies. The Europe 2020 strategy sets the target of 3\% GDP for R\&D spending. Many studies emphasize a significant effect of increasing expenditures on $R \& D$ on economic growth, but an efficiency aspect has not been covered in the literature. The article is based on critical review of the main literature of the subject and own empirical studies. The statistical data is sourced from the main international statistics. Calculations were performed using DEA methodology. DEA methodology allows assessing input-output efficiency. Inputs indicator is the annual public and private spending on $R \& D$ (as \% GDP). There are nine output indicators, which represent available innovative statistics about number of patents, high-tech production etc. Number of variables was reduced for each period using correlation coefficient analysis, which allowed identifying the significant variables with least loss of information. The efficiency is calculated as the ratio of the weighted sum of the outputs by the weighted sum of inputs. The calculations are carried out based on the Excel spreadsheet and DEAFrontier. The paper gives a general review of the innovation level in CEE countries compared to other EU members which are spending less than $2 \%$ of GDP on $R \& D$. The analysis shows that among CEE countries, the closest to efficiency frontier are Romania and Slovakia. Hypothesis that increasing spending on innovations is not causing proportional effects has been confirmed for CEE region, but not for western economies, which are spending on $R \& D$ more effectively. Main conclusion of the research is that innovation spending should be increased gradually in aim to achieve optimal results. This research may contribute to discussion on innovation policy design, and can be used by policy makers to develop national innovation strategies.
\end{abstract}

Key words: innovation, DEA methodology, relative efficiency, investment

JEL classification: H50, O30, O38, O57, R15

\footnotetext{
* Received: 02-05-2018; accepted: 13-12-2018

1 Assistant Professor, Wroclaw University of Economics, Faculty of Economics, 118/120 Komandorska St, 53-345 Wroclaw, Poland. Scientific affiliation: international economics. Phone: +48601594346,E-mail: pawel.dobrzanski@ue.wroc.pl
} 


\section{Introduction}

Innovativeness of the economy is one of the key factors stimulating economic growth. Many countries have an increased focus on developing the innovation policy and strategy for their countries. Improving innovativeness is especially important for developing countries, which are trying to improve their competitiveness and stimulate economic growth. Many of the Member States of the European Union are among the most innovative and developed economies in the World. In 2004 ten post-transition countries joined the European Union from Central and Eastern Europe. Since then, a concept of two speed Europe competitive and innovative old Members, and lower-income new Member States has become a popular topic in the literature.

Innovation policy is important for the European Union. R\&D spending in Europe in 2010 was below $2 \%$ of GDP, while in the USA it was $2.6 \%$ and in Japan $3.4 \%$. This was result of lower levels of private investment. European Commission is emphasising that there is a strong need to improve conditions for private $R \& D$ in the EU. EU policymakers even set up a goal in the Europe 2020 Strategy to ensure that $3 \%$ of GDP should be invested in R\&D in all Members States. As fiscal policy is likely to remain under pressure in many Members States, public expenditures probably won't increase significantly. Therefore, to reach 2020 target business expenditure should increase by 80\% (Gros, Roth,2012). "Innovation Union" was one of the initiatives created to improve conditions and access to finance for research and innovation. The Commission launched it to support regions and Member States in better defining their innovation strategies by assessing their innovative strengths and weaknesses and build on their competitive advantage (European Commission, 2013a). However, despite all those activities, it is doubtful that $3 \%$ GDP goal will be achieved. EU's spending on R\&D over last years remains almost at the same level around $2 \%$ of GDP.

Another question that can arise, is whether the level of 3\% of GDP spending on R\&D is worthwhile and optimal for all Member Countries. Even in the Europe 2020 Strategy it is stated that the target focuses on input rather than output. For EU policy makers it is clear that analysing $R \& D$ and innovation together would get more relevant productivity drivers. Finally, the Commission proposed to keep the $3 \%$ target, while developing an indicator which would reflect R\&D and innovation intensity (European Commission, 2010).

The aim of the study is to verify if money spend on $R \& D$ are used efficiently in CEE countries. Main hypothesis of the research is that increasing $R \& D$ spending is not causing proportional innovative effects.

The paper is organized as follows. Second section presents literature review regarding innovation in economic growth concept and innovation policies. Section 
three describes DEA methodology. Section four presents data chosen for analysis. Section five contains research findings and the last section concludes the research.

\section{Literature review}

According to new growth theories long-term economic growth can be achieved endogenously thanks to innovation and technological progress. The significance of innovation for economic growth has been emphasized by many economists. Concept of innovation was introduced by Schumpeter, who declared that, innovative companies stimulate economic development and competitiveness by "creative destruction". Innovations replace old products and technologies, having a positive impact on the turnover outcome (Schumpeter, 2003). Gartner and Drucker (1987) defined innovation as instrument of entrepreneurship, which creates new opportunities to generate wealth. Solow (1956) stated that technological change, rather than capital accumulation, is the main driver of long-run growth. Romer (1986) underlined importance of innovation and entrepreneurship in stimulating economic growth. Also, some empirical researches are confirming positive impact of innovation on economic growth (Fagerberg and Srholec, 2008; Hirooka, 2006; Taylor, Grossman and Helpman, 1993). Therefore, innovation is nowadays at the center of each competitiveness strategy, both company as well as government policies.

Literatures underline also the relationship between innovation and regulation. Companies must have the willingness, opportunity, motivation, and capability or capacity to innovate. Policy regulations can cause both positive and negative impact (Carlin and Soskice, 2006). EU regulation matters at all stages of the innovation process, from R\&D to commercialization. Regulation can be a powerful incentive for innovative actions, but at times regulation can disable innovation. The impact of the regulation on innovation depends on the balance between innovation-inducing factors and compliance costs generated by legal provision (Pelkmans, Renda, 2014).

The aim of European Union is to improve its competitiveness through innovation. The changing global landscape and the growing importance of innovation require EU to review its innovation policy, including both the scope and the governance of innovation at the EU and national level. Policymakers should consider the whole innovation cycle, including all links in the innovation chain: industry, university, public and private financing organizations, society, politicians, policymakers etc. The innovation policy should consider both the supply and the demand for innovation. For that reason, the European Institute of Innovation and Technology (EIT) was established in 2007. The EIT is the first European initiative that aims to integrate knowledge triangle, which consists Higher Education, Research and 
Business-Innovation. EIT objective is to improve innovation capacity and capability of the EU. However, still there is no standard policy implemented in all Member States. Innovative goals are announced at the EU level and Member States have full flexibility in their implementation. From one hand codified policy may simplify the sharing of common technological solutions, removing trade barriers, enabling technology transfer, and boosting the creation of complementary markets. But, in some cases it can lead to undesirable results (Anvret, Granieri, Renda, 2010).

The European Commission launched its innovation goals in the Europe 2020 Strategy, which announces seven flagship initiatives, of which at least five are closely linked with innovation (Innovation Union, Digital Agenda, Resource Efficient Europe, A New Industrial Policy for the new globalization era and an Agenda for new skills and jobs). Main goal of this strategy is to achieve smart, sustainable and inclusive growth, improve its competitiveness and productivity and underpin a sustainable social market economy. This should be guarantee by 3\% GDP spending on R\&D (European Commission, 2010). The European Council agreed that progress towards the Innovation Union should be measured at EU level and only R\&D indicator is not reflecting full picture of countries innovativeness. Therefore, the European Council organized high-level panel with leading business innovators and economists to identify possible indicators, which would best assess innovation intensity. New indicator should have focus on outputs and impacts and facilitate international comparability. Moreover, the European Council underlined urgent need of improving data availability and quality to measure and monitor innovation performance. The Commission also emphasized that, because innovation is a multi-faceted phenomenon, further work is needed to develop indicators on aspects such as non-technological innovation, design, service innovation, and performance at regional level (European Commission, 2011).

Both policy makers and researchers agreed that the R\&D spending indicator had certain limitations and is not correctly accessing innovative improvements of the Member States. The use of R\&D spending as innovative indicator is widely criticized in the literature. Tilford and Whyte (2010) pointed out that EU should not neglect $\mathrm{R} \& \mathrm{D}$, but move beyond focus on numerical $\mathrm{R} \& \mathrm{D}$ targets and provide the broader concept of innovation. Moreover, $R \& D$ is concentrating mainly on the manufacturing sector, omitting service sector. EU is service-dominated economy, with the highest share of service sector in GDP, which is several times bigger than that of manufacturing. Gros and Roth (2012) also emphasized that European Union should utilise the broader concept of innovation, named concept of intangible capital, which would align better with economic structure of EU. Intangible capital concept includes capital, which is not included in national accounts, such as: architectural design, new financial products, own-account and purchased organizational structure of a firm, firm-specific human capital, branding, market research and scientific R\&D. Zabala-Itturiagagoitia (2008) has indicated 
that referring only to input indicators might result in overrating unproductive $R \& D$ investment. Most of public R\&D is used not to stimulate economic growth, but to achieve public agency goals and any contribution to economic growth is thus due to indirect knowledge transfers.

In 2013, the European Commission presented a new indicator to capture innovation outputs, which can be used for measuring the EU's progress in meeting the goals of the Europe 2020 Strategy. Aim of the new indicator is to support policy-makers in establishing policies to remove barriers that prevent innovators from transforming ideas into successful products and services. The Indicator of Innovation Output combines four output sub-indicators. First is technological innovation measured by number of patents. This indicator takes into account knowledge generated by investing in R\&D that can be transformed into successful technologies. Second indicator, employment in knowledge-intensive activities as a percentage of total employment, provides an economic orientation towards the production of goods and services with innovation added value. Third indicator, competitiveness of knowledge-intensive goods and services, is based on the share of high-tech and medium-tech products trade in the total trade balance, and share of knowledge-intensive services in the total services trade. Last indicator is employment in fast-growing firms of innovative sectors. Stimulating jobs in such sectors is integral part of modern research and innovation policy. This indicator provides a measure of the dynamism of the economy, capturing relation between growth and jobs (European Commission, 2013b).

However, new innovation indicator solves only one problem. It supplements the input perspective with an output perspective, but it tends to disregard actual innovation outcomes. Enterprises can transform innovation inputs, such as R\&D, human resources, research infrastructures and existing knowledge, in a first stage into intermediate outputs, such as patents, and in a second stage, into innovation outcome. Innovation outcomes are the results of the introduction of innovations, among them the economic effects of innovation outputs on firms introducing them. Patent application itself does not automatically have economic results. For adequately measuring innovation outcomes at the country level, both structural change and structural upgrading should be considered. Structural change is reallocation economic activity towards more knowledge-intensive sectors. Structural upgrading is getting closer to the frontier in sectors countries are already specialised in. This is related to differential performance of enterprises without necessarily changing the overall composition of the economic structure, e.g. by moving to more knowledge-intensive activities within the same sector (Janger et al., 2017). Unfortunally, statistics for innovative outcomes are not conducted, due to difficulty in obtaining realiable data.

Innovativeness and innovative strategies and policies are widely described in the economic literature. Most publications are focusing mainly only on inputs defined in innovative policies or outputs achieved by countries or regions. DEA efficiency 
of R\&D spending for countries or regions is less frequently discussed topic. Such an approach is caused by both problems in defining efficiency and measuring it. There are various studies elaborating innovation efficiencies both on micro or macro level. Cruz-Cazares et al. (2013) performed empirical analysis for Spanish manufacturing firms for the period 1992-2005. Inputs variables were R\&D capital stock and high-skill staff and two outputs of the innovation process that account for the number of product innovations and the number of patents were selected for analysis. Technological innovation efficiency was calculated with DEA methodology. Study also confirmed positive relationship between R\&D efficiency and firm performance. Hashimotoa and Hanedab (2008) used DEA/Malmquist index analysis to examine time series change in R\&D efficiency at industry level for 10 Japanese pharmaceutical firms for decade 1983-1992. The Malmquist index was used to measure the ratio of DEA efficiencies in two different time periods with shifting DEA efficiency frontiers. There are also many regional studies. Guan and Zuo (2017) evaluated R\&D efficiency of the 30 provinces of China with three different methods: CRS, Cross-efficiency and Game Cross-efficiency. They employed fulltime equivalent researchers and expenditure on R\&D as inputs variables and number of granted patents and publication in scientific journals as the output variables. Broekel et al. (2017) used shared-input DEA-model to compute regions' innovation efficiency for 150 German regions. They constructed an allindustry regional innovation efficiency measure that explicitly considers interregional variations in regions' industrial structures. Moreover, regional studies of $\mathrm{He}$ et al. (2018) and Liu et al. (2018) confirmed spatial dependence of R\&D efficiency, and existence of spillover effect. There are also numerus publications that are assessing efficiency of countries. Sharma and Thomas (2008) examined the relative efficiency of the R\&D process across a group of 22 developed and developing countries under CRS and VRS framework. Inputs variables selected for analysis were Gross Domestic Expenditure on Research and Development in Million Dollars (PPP) and the number of researchers per million inhabitants. The outputs of the R\&D process were measured by number of patents and publications. The data on inputs was collected for the year 2002 and on outputs for 2004 . Research included both developed and developing countries, but only those with R\&D expenditure above $0.75 \%$ of GDP. Guan and Chen (2012) divided innovation processes into knowledge production process and knowledge commercialization process and applied dual network-DEA models for 22 countries. Guan and Zuo (2014) used the same dual approach for 35 countries over the period 2007-2011. Their research not only estimated technical efficiency and scale efficiency for each country, but also verified whether returns-to-scale of each country are decreasing or increasing. They also highlighted the importance of time lag between inputs and outputs. Chen et al. (2013) analysed 29 Countries for the period 1998-2005 using Luenberger productivity index. This study decomposed the LRC index into two modules: change in relative R\&D efficiency (measuring catch-up effect or fallbehind effect) and shift in the production frontier under the total-factor framework. 
In all mentioned studies different input and output variables were selected, which makes each research unique and provides different conclusions. Moreover, in all researches results depends on number of variables and used methodology. The choice of input and output of DEA methodology has a critical impact on the result of efficiency measurement. Measuring technical innovation efficiency is not new topic in economic analysis, however, empirical evidence is still limited and need to be supplemented and developed. The contributions of this paper to existing literature are as follows. First, this article provides a study of R\&D efficiency for CEE countries in comparison to other EU member states. Improving efficiency is especially important for developing countries, which justify selection of CEE countries for additional analysis. Furthermore, Author didn't find R\&D efficiency studies published for CEE or EU countries and analysis attached in the paper can fill in this gap in literature. Second, analysed will be period of the latest available data for years 2008-2015. Choosing such period for research allowed verifying efficiency trend for analysed economies.

\section{Methodology}

Methodology employed in the research is Data Envelopment Analysis (DEA). DEA is the nonparametric methodology used for efficiency measurement. The precursor of this methodology was Farrell (1957) and it was further developed by Charnes et. al. (1978). DEA measures the efficiency of units with multiple outputs and multiple inputs along with objectively determining weights. Equivalences are established to ordinary linear programming models for effecting computations. (Charnes et. al. 1978). DEA is a methodology for measuring comparative, relative and so-called technical efficiency. Efficiency is relative, as it measures efficiency with reference to some set of units that are being compared with each other. In general terms, the essential idea is to assess how efficiently each decision-making unit (DMU) is handling the transformation process when compared to other DMUs engaged in the same process. To do this relation between outputs achieved and available resources is analyzed. DEA is not absolute measure of efficiency. Units which are efficient in DEA methodology may in fact be capable of improving their performance even further. The DEA model is an input-oriented model, which seeks to identify technical efficiency as a proportional reduction in input usage (Thanassoulis, 2001). The efficient DMUs are not necessarily production frontier, but rather best-practice frontier (Cook, Tone and Zhu, 2014).

DEA can separate the efficient operating units from the inefficient on the basis of whether they lie on the efficient frontier which is spanned by the best units in a data set. The efficiency measure employed in DEA is established mathematically by the ratio of the weighted sum of outputs to the weighted sum of inputs (Cooper et al., 2007): 


$$
\text { Effectiveness }=\frac{\sigma_{r=1}^{R} u_{r} y_{r j}}{\sigma_{N=1}^{N} v_{n} x_{n j}},
$$

where:

$u_{r}$ - weight of output

$v_{n}$-weight of input

$y_{r j}-$ output

$x_{n j}$ input

The first step in the application of DEA is to agree on relevant inputs and outputs. Inputs and outputs do not have to be measured in the same units. In DEA the resources are typically referred as inputs and the outcomes as outputs. Identification of the input-output variables used in an assessment is the most crucial step. The results, which will be obtained in the research depend crucially on the choice made. The input-output variables are unique to the type of efficiency being assessed. The inputs should capture all resources, which impact the outputs and the outputs should reflect all useful outcomes, on which we wish to assess the DMUs. The identification of exogenous variables is important. Exclusivity and exhaustiveness of input-output variables must guide the choice of the input-output variables subject to the exogeneity of any variables being proposed. (Thanassoulis, 2001) With DEA methodology the overall efficiency of a DMU is measured by its total factor productivity output-to-input ratio, which takes into account all outputs and all inputs. The main problem here is choosing the inputs and outputs to be considered and the weights to be used in order to obtain a complex overall measure (Wober, 2007). It is necessary to remove output data, which can duplicate information (Jenkins and Anderson, 2003). In presented analysis Pearson's linear correlation coefficient between variables was used (DeVolpi, 1991):

$$
r=\frac{\operatorname{cov}\left(X_{i} Y_{i}\right)}{s_{i} S_{j}}
$$

where:

$\operatorname{cov}\left(X_{i} Y_{i}\right)$ - covariance between i-variable and j-variable

$s_{i}$ - standard deviation of variable $X_{i}$

$s_{j}-$ standard deviation of variable $X_{j}$.

As many research methods, DEA has advantages and disadvantages. Thanassoulis (2003) underlined that DEA methodology has many benefits. First of all, there is no need to specify a mathematical function of the efficiency. Moreover, DEA methodology can be useful in uncovering relationships that remain hidden for other 
methodologies. DEA methodology allows analysing multiple inputs and outputs at the same time, without any input-output measurement. In addition, the sources of inefficiency can be analysed and quantified for every evaluated unit. Wober (2007) also underlined that DEA needs no a priori information regarding which inputs and outputs are the most important in the evaluation procedure. This gives possibility to use it for analysis for complex and often unknown nature of relationships between variables. The model implies that inputs and outputs are measurable, and infinitely divisible. DEA does not consider qualitative information and some crucial factors affecting efficiency could not be included into analysis. Therefore, careful interpretation and sensitivity analysis is required. DEA can be a useful and powerful methodology of analysis for someone, who fully understands both its potential and its limitations (Molinero and Woracker, 1996).

In the research presented in this article, few DEA limitations can be indicated. First, the choice of inputs and outputs has a decisive influence on the result of efficiency measurement. Data for analysis was chosen based on their availability from international statistics. Secondly, initially only CEE economies were selected for analysis, which gives small number of DMUs. To increase number of DMUs additional EU countries were selected for analysis. Author has chosen EU countries, which in the analysed period did not spend more than $2 \%$ of GDP on R\&D. This allowed obtaining a comparable peer group. Thirdly, if there exist a large number of input and output variables, the discriminatory power of the DEA will reduce. In the conducted research initial list of variables contained 1 input and 9 output variables. Moreover, some output data may duplicate information or coincidence may exist between the inputs and outputs variable. For that reason, number of variables was reduced for each period using correlation coefficient analysis. This allowed identifying the significant variables with least loss of information. Finally, the DEA is only an assessment of relative efficiency, and can't replace absolute efficiency. This means, that results present efficient economies only for selected group of 20 countries. If the group would be expanded, effective individuals may turn out to be inefficient.

\section{Empirical data and analysis}

The aim of the study is to examine the relationship between innovation expenditure (input) and the innovation results achieved. Excel Spreadsheet and DEAFrontier were used for calculations. The study was limited to $20 \mathrm{EU}$ countries and periods of research from 2008 to 2015. Apart from the countries from the CEE region, study included also EU countries, which in the analyzed period did not spend more than $2 \%$ of GDP on R\&D. This allowed to increase the amount of DMUs and to obtain a comparable peer group. The research employed Data Envelopment Analysis (DEA) methodology. The efficiency in DEA is defined as the ratio of the weighted sum 
of the results by the weighted sum of inputs. Performance is relative, because it is created in relation to the entire group of objects.

The estimated efficiency will indicate to what extent expenditure on innovation affected efficiency in selected countries. Selection of diagnostic variables was carried out based on available statistical data. The nine output indicators chosen for analysis are as follows. First European Union trade mark as a applications per million population (EUTM), second one is high-technology exports as a percentage of manufactured exports, than employment in high- and medium-high technology manufacturing sectors as a percentage of total employment, human resources in science and technology (HRST) as a percentage of active population, patent applications to the European patent office (PA) by priority year for mln inhabitants, high-tech patent applications to the European Patent Office (PA-HT) by priority year for mln inhabitants, graduates in tertiary education, in science, math., computing, engineering, manufacturing, construction per 1000 of population aged 20-29 (GTE), employment in knowledge-intensive service sectors as a percentage of total employment (ETH-S), scientific and technical journal articles for mln inhabitants (ATJA). Data was sourced from the Eurostat database and the World Bank. In DEA methodology anti-stimulants must be converted using differential formula to stimulants. In analyzed case, all the output variables are stimulants.

Table 1: Indicators and sources

\begin{tabular}{|c|c|c|c|}
\hline Variable & Full indicator name & Units & Source \\
\hline RDE & $\begin{array}{l}\text { The annual public and private spending on } \\
\text { innovation }\end{array}$ & (as \% GDP) & Eurostat \\
\hline $\mathrm{PA}$ & $\begin{array}{l}\text { Number of patent applications to the European } \\
\text { patent office by priority year }\end{array}$ & (Per mln inhabitants) & Eurostat \\
\hline ATJA & Scientific and technical journal articles & (Per mln inhabitants) & World Bank \\
\hline HRST & Human resources in science and technology & $\begin{array}{l}\text { (\% of active population - } \\
\text { from } 25 \text { to } 64 \text { years })\end{array}$ & Eurostat \\
\hline PA-HT & $\begin{array}{l}\text { High-tech patent applications to the European } \\
\text { patent office by priority year }\end{array}$ & (Per mln inhabitants) & Eurostat \\
\hline EUTM & European Union trade mark applications & (Per million inhabitants) & Eurostat \\
\hline EHT-S & $\begin{array}{l}\text { Employment in knowledge-intensive service } \\
\text { sectors }\end{array}$ & (\% of total employment) & Eurostat \\
\hline EHT-M & $\begin{array}{l}\text { Employment in high- and medium-high } \\
\text { technology manufacturing sectors }\end{array}$ & (\% of total employment) & Eurostat \\
\hline HTE & Exports of high-tech products & (\% of exports) & Eurostat \\
\hline GTE & $\begin{array}{l}\text { Graduates in tertiary education, in science, } \\
\text { math, computing, engineering, manufacturing, } \\
\text { construction }\end{array}$ & $\begin{array}{l}\text { Per } 1000 \text { of population } \\
\text { aged } 20-29\end{array}$ & Eurostat \\
\hline
\end{tabular}

Source: Eurostat and World Bank Data Base 
Pawel Dobrzanski • Innovation expenditures efficiency in Central and Eastern...

Inputs indicator are the annual public and private spending on innovation (as \% GDP) represented by RDE. Source of this data is Eurostat Database. Due to the lack of available data some indicators are marked with "*”, where values are taken from the period preceding, following or their average. The list of all variables is presented in the table 1. In table 2 Author has presented input and output data for EU20 in 2008. The data for other periods (2009-2015) has been presented in the appendix.

Table 2: Diagnostic data of inputs and outputs - EU20 in 2008

\begin{tabular}{|l|r|r|r|r|r|r|r|r|r|r|}
\hline $\begin{array}{c}\text { Country/ } \\
\text { Indicators } \\
(2008)\end{array}$ & RDE & PA & ATJA & HRST & PA-HT & EUTM & EHT-S & EHT-M & HTE & GTE \\
\hline Bulgaria - CEE & 0,45 & 2,48 & 314,51 & 31,4 & 0,509 & 18,75 & 27,2 & 4,2 & 3,6 & 9,6 \\
\hline Czechia - CEE & 1,24 & 20,27 & 1025,47 & 37,1 & 2,424 & 36,74 & 29,7 & 10,2 & 14,1 & 15,2 \\
\hline Estonia-CEE & 1,26 & 26,4 & 836,14 & 44,4 & 13,038 & 67,99 & 31,3 & 4 & 7,5 & 12 \\
\hline Ireland & 1,39 & 73,66 & 1288,93 & 43,4 & 20,122 & 156,58 & 40,2 & 4,7 & 24,3 & 18,8 \\
\hline Greece & 0,66 & 8,51 & 1062,85 & 31,9 & 1,304 & 35,71 & 32,5 & 1,7 & 4,9 & 10,7 \\
\hline Spain & 1,32 & 31,61 & 956,58 & 38,9 & 5,678 & 141,17 & 30,9 & 4 & 4,2 & 11,5 \\
\hline Croatia-CEE & 0,88 & 6,70 & 896,69 & 29,0 & 1,895 & 4,4 & 27,1 & 3,8 & 6,7 & 10,7 \\
\hline Italy & 1,16 & 81,04 & 950,94 & 35,4 & 7,851 & 118,29 & 33,6 & 6 & 5,9 & 11,7 \\
\hline Cyprus & 0,39 & 14,49 & 428,45 & 43,7 & 1,288 & 185,49 & 34,5 & 0,8 & 19,1 & 4,2 \\
\hline Latvia-CEE & 0,58 & 10,39 & 268,82 & 39,4 & 1,369 & 21,90 & 32,2 & 1,9 & 4,6 & 9,4 \\
\hline Lithuania-CEE & 0,79 & 5,25 & 779,49 & 42,3 & 1,827 & 24,59 & 30,6 & 2,1 & 6,5 & 20,2 \\
\hline Luxembourg & 1,62 & 193,41 & 633,79 & 45,5 & 10,748 & 1544,03 & 54,2 & 0,9 & 35,6 & 3 \\
\hline Hungary-CEE & 0,98 & 18,04 & 638,41 & 33,3 & 4,55 & 16,13 & 33,2 & 8,6 & 20,2 & 6,1 \\
\hline Malta & 0,53 & 13,49 & 432,36 & 32,2 & 4,904 & 154,48 & 39,7 & 4,8 & 38,3 & 6,1 \\
\hline Netherlands & 1,64 & 210,7 & 1613,47 & 51,1 & 49,314 & 184,57 & 46,9 & 3,1 & 16,2 & 8,8 \\
\hline Poland-CEE & 0,6 & 6,1 & 613,13 & 33,4 & 0,888 & 23,72 & 28,3 & 5,4 & 4,3 & 14,1 \\
\hline Portugal & 1,45 & 11,09 & 819,80 & 23,0 & 2,729 & 72,58 & 28,4 & 3 & 6,3 & 17 \\
\hline Romania-CEE & 0,57 & 1,62 & 358,74 & 23,8 & 0,662 & 9,40 & 19 & 5 & 5,4 & 17,9 \\
\hline Slovakia-CEE & 0,46 & 6,86 & 619,51 & 32,0 & 1,581 & 17,48 & 29,6 & 10,2 & 5,2 & 15,2 \\
\hline $\begin{array}{l}\text { United } \\
\text { Kingdom }\end{array}$ & 1,63 & 87,56 & 1488,51 & 43,7 & 18,808 & 130,95 & 46,2 & 4,7 & 15,4 & 19,1 \\
\hline $\begin{array}{l}\text { Average for } \\
\text { CEE }\end{array}$ & 0,78 & 10,41 & 635,09 & 34,61 & 2,87 & 24,11 & 28,82 & 5,54 & 7,81 & 13,04 \\
\hline $\begin{array}{l}\text { Average for } \\
\text { EU20 }\end{array}$ & 0,98 & 41,48 & 801,33 & 36,75 & 7,57 & 148,25 & 33,77 & 4,46 & 12,42 & 12,07 \\
\hline
\end{tabular}

Source: Author's own study based on Eurostat and World Bank Data Base 
Number of variables was reduced for each period using correlation coefficient analysis. This allowed to remove indicators, which duplicate information and identify the significant variables with least loss of information. Information replicate variables are highly correlated (correlation coefficient $>0.7,(p<0.05))$. To obtain the accuracy of the model three of these variables were removed for 2008 (PA, HTE, EHT-S). This relationship is shown in the table 3.

Table 3: Correlation coefficients -2008

\begin{tabular}{|l|c|c|c|c|c|c|c|c|c|c|}
\hline & RDE & PA & ATJA & HRST & PA-HT & EUTM & EHT-S & EHT-M & HTE & GTE \\
\hline RDE & 1,000 & & & & & & & & & \\
\hline PA & 0,712 & 1,000 & & & & & & & & \\
\hline ATJA & 0,751 & 0,548 & 1,000 & & & & & & & \\
\hline HRST & 0,458 & 0,658 & 0,459 & 1,000 & & & & & & \\
\hline PA-HT & 0,654 & 0,814 & 0,734 & 0,659 & 1,000 & & & & & \\
\hline EUTM & 0,413 & 0,675 & $-0,018$ & 0,377 & 0,179 & 1,000 & & & & \\
\hline EHT-S & 0,593 & 0,839 & 0,459 & 0,716 & 0,644 & 0,696 & 1,000 & & & \\
\hline EHT-M & $-0,050$ & $-0,234$ & 0,054 & $-0,289$ & $-0,117$ & $-0,358$ & $-0,286$ & 1,000 & & \\
\hline HTE & 0,256 & 0,472 & 0,048 & 0,352 & 0,289 & 0,606 & 0,725 & $-0,084$ & 1,000 & \\
\hline GTE & 0,146 & $-0,262$ & 0,338 & $-0,190$ & $-0,024$ & $-0,452$ & $-0,356$ & 0,265 & $-0,457$ & 1,000 \\
\hline
\end{tabular}

Source: Authors' calculations

In DEA methodology, in oppose to the statistical methods, strong correlation is unwelcome. Another assumption in DEA model is coincidence between the inputs and outputs variable. For that reason, correlation coefficient between inputs and outputs was verified. Output variables with positive correlation with input variable can remain in the model. In analyzed case for 2008, one output variable was removed (EHT-M). Final set of features is presented in table 4. 
Pawel Dobrzanski • Innovation expenditures efficiency in Central and Eastern...

Table 4: The final set of features inputs and outputs - EU20 in 2008

\begin{tabular}{|l|r|r|r|r|r|r|}
\hline Country/Indicators (2008) & \multicolumn{1}{c|}{ RDE } & \multicolumn{1}{c|}{ HRST } & PA-HT & \multicolumn{1}{c|}{ EUTM } & HTE & \multicolumn{1}{c|}{ GTE } \\
\hline Bulgaria - CEE & 0,45 & 31,4 & 0,509 & 18,75 & 3,6 & 9,6 \\
\hline Czechia - CEE & 1,24 & 37,1 & 2,424 & 36,74 & 14,1 & 15,2 \\
\hline Estonia - CEE & 1,26 & 44,4 & 13,038 & 67,99 & 7,5 & 12 \\
\hline Ireland & 1,39 & 43,4 & 20,122 & 156,58 & 24,3 & 18,8 \\
\hline Greece & 0,66 & 31,9 & 1,304 & 35,71 & 4,9 & 10,7 \\
\hline Spain & 1,32 & 38,9 & 5,678 & 141,17 & 4,2 & 11,5 \\
\hline Croatia-CEE & 0,88 & 29,0 & 1,895 & 4,4 & 6,7 & 10,7 \\
\hline Italy & 1,16 & 35,4 & 7,851 & 118,29 & 5,9 & 11,7 \\
\hline Cyprus & 0,39 & 43,7 & 1,288 & 185,49 & 19,1 & 4,2 \\
\hline Latvia-CEE & 0,58 & 39,4 & 1,369 & 21,90 & 4,6 & 9,4 \\
\hline Lithuania-CEE & 0,79 & 42,3 & 1,827 & 24,59 & 6,5 & 20,2 \\
\hline Luxembourg & 1,62 & 45,5 & 10,748 & 1544,03 & 35,6 & 3 \\
\hline Hungary-CEE & 0,98 & 33,3 & 4,55 & 16,13 & 20,2 & 6,1 \\
\hline Malta & 0,53 & 32,2 & 4,904 & 154,48 & 38,3 & 6,1 \\
\hline Netherlands & 1,64 & 51,1 & 49,314 & 184,57 & 16,2 & 8,8 \\
\hline Poland - CEE & 0,6 & 33,4 & 0,888 & 23,72 & 4,3 & 14,1 \\
\hline Portugal & 1,45 & 23,0 & 2,729 & 72,58 & 6,3 & 17 \\
\hline Romania-CEE & 0,57 & 23,8 & 0,662 & 9,40 & 5,4 & 17,9 \\
\hline Slovakia - CEE & 0,46 & 32,0 & 1,581 & 17,48 & 5,2 & 15,2 \\
\hline United Kingdom & 1,63 & 43,7 & 18,808 & 130,95 & 15,4 & 19,1 \\
\hline
\end{tabular}

Source: Authors' calculations

Finally, efficiency was calculated using DEAFrontier. The results of these calculations have been collected in table 5 for CRS and VRS in table 6. There are several types of DEA models which can be used. In analyzed case Author used two input-oriented models: CRS (constant returns-to-scale) and VRS (variable returnsto-scale). The CRS reflects the fact that output will change by the same proportion as inputs are changed. On the other hand, VRS reflects the fact that production technology can exhibit increasing, constant and decreasing returns to scale. Author has chosen Input-oriented model to test if a DMU under evaluation can reduce its inputs while keeping the outputs at their current levels. 


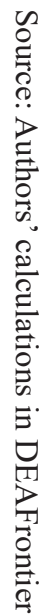

\begin{tabular}{|c|c|c|c|c|c|c|c|c|c|c|c|c|c|c|c|c|c|c|c|c|}
\hline$\tilde{\circ}$ & $\overrightarrow{0}$ & $\infty$ & $\Xi$ & $\vec{a}$ & $\vec{u}$ & $\Phi$ & $\vec{\omega}$ & $\vec{N}$ & 二 & $\sigma$ & 0 & $\infty$ & $\checkmark$ & $a$ & $u$ & $\triangle$ & $\omega$ & $N$ & - & Z๐ \\
\hline 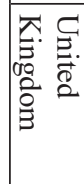 & 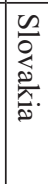 & 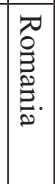 & 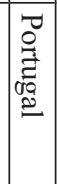 & $\begin{array}{l}0 \\
0 \\
20 \\
0 \\
0\end{array}$ & 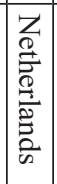 & \begin{tabular}{|l|}
3 \\
\multirow{2}{*}{} \\
2 \\
\end{tabular} & 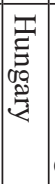 & 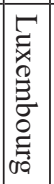 & 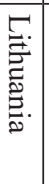 & 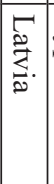 & 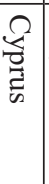 & 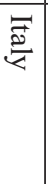 & 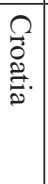 & 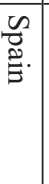 & $\begin{array}{l}0 \\
0 \\
0 \\
0 \\
0 \\
0\end{array}$ & $\begin{array}{l}\vec{F} \\
\stackrel{0}{0} \\
\stackrel{0}{2} \\
\overrightarrow{2}\end{array}$ & 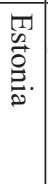 & 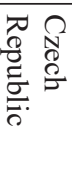 & 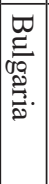 & 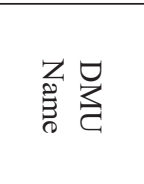 \\
\hline$\stackrel{\circ}{2}$ & 5 & 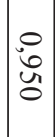 & $\mid \begin{array}{l}0 \\
\stackrel{0}{0} \\
\stackrel{N}{N}\end{array}$ & \begin{tabular}{l}
0 \\
\hdashline \\
$\vdots$ \\
$\vdots$
\end{tabular} & : & $\mid \begin{array}{l}- \\
8 \\
8\end{array}$ & $\begin{array}{l}0 \\
\dot{\omega}=1 \\
0 \\
0\end{array}$ & 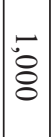 & \begin{tabular}{l}
0 \\
\multirow{2}{a}{} \\
$\sigma$
\end{tabular} & $\mid \begin{array}{l}0 \\
\stackrel{0}{+} \\
\dot{4}\end{array}$ & 5 & $\begin{array}{l}0 \\
\stackrel{0}{a} \\
\dot{0}\end{array}$ & 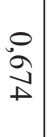 & $\begin{array}{l}0 \\
\ddot{\varkappa} \\
2\end{array}$ & 5 & $\begin{array}{l}0 \\
\infty \\
u_{1} \\
u\end{array}$ & $\begin{array}{l}0 \\
\stackrel{0}{a} \\
\sigma\end{array}$ & $\begin{array}{l}0 \\
\text { Nิ } \\
\text { Na }\end{array}$ & $\mid \begin{array}{c}0 \\
\infty \\
w \\
+ \\
+\end{array}$ & 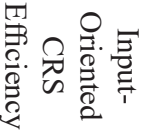 \\
\hline 崩 & : & $\stackrel{\square}{\vdots}$ & w & : & 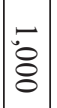 & $\mid \begin{array}{l}- \\
8 \\
8 \\
8\end{array}$ & 5 & : & $\underset{\breve{w}}{\sim}$ & $\mid$ & 5 & 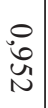 & $\begin{array}{l}0 \\
\text { o } \\
0 \\
0\end{array}$ & $\begin{array}{l}\text { 芯 } \\
\text { న̀ }\end{array}$ & 5 & $\begin{array}{l}5 \\
\dot{a} \\
\dot{u}\end{array}$ & $\frac{n}{\sigma}$ & ư & $\mid \begin{array}{l}0 \\
\infty \\
\infty \\
\vdots \\
\end{array}$ & $>\circ \stackrel{\Xi}{E}$ \\
\hline 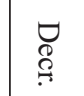 & 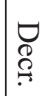 & 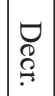 & $\begin{array}{l}0 \\
\varnothing \\
\stackrel{9}{9}\end{array}$ & $\begin{array}{l}\underset{\infty}{0} \\
\stackrel{9}{9}\end{array}$ & 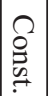 & 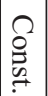 & $\begin{array}{l}\underset{\otimes}{0} \\
\stackrel{9}{9}\end{array}$ & 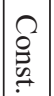 & 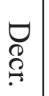 & \begin{tabular}{|l|} 
\\
$\varnothing$ \\
$\stackrel{9}{9}$
\end{tabular} & $\begin{array}{l}\stackrel{\Omega}{0} \\
\stackrel{2}{n}\end{array}$ & 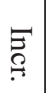 & 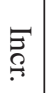 & 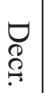 & 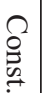 & $\nabla$ & 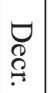 & 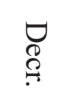 & 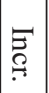 & $\tau$ \\
\hline
\end{tabular}

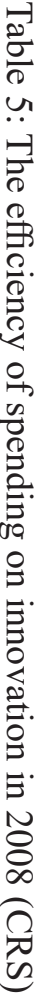

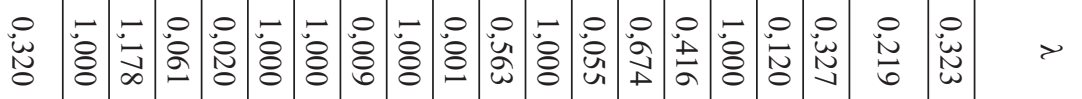

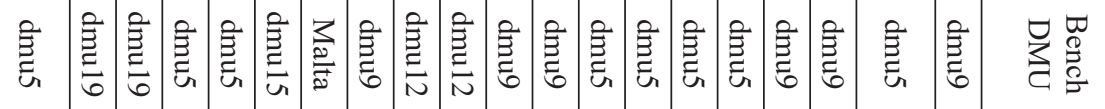

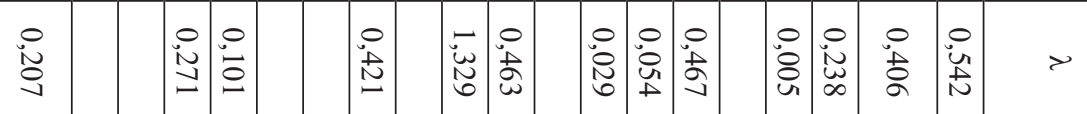

䒠

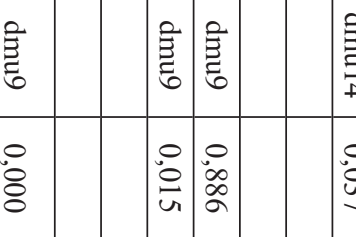

\begin{tabular}{|c|c|c|}
\hline$\underset{\text { 竔 }}{\text { N }}$ & 羙 & 语 \\
\hline$\stackrel{0}{\tilde{\omega}}$ & $\begin{array}{l}0 \\
0 \\
0 \\
0\end{array}$ & \\
\hline
\end{tabular}

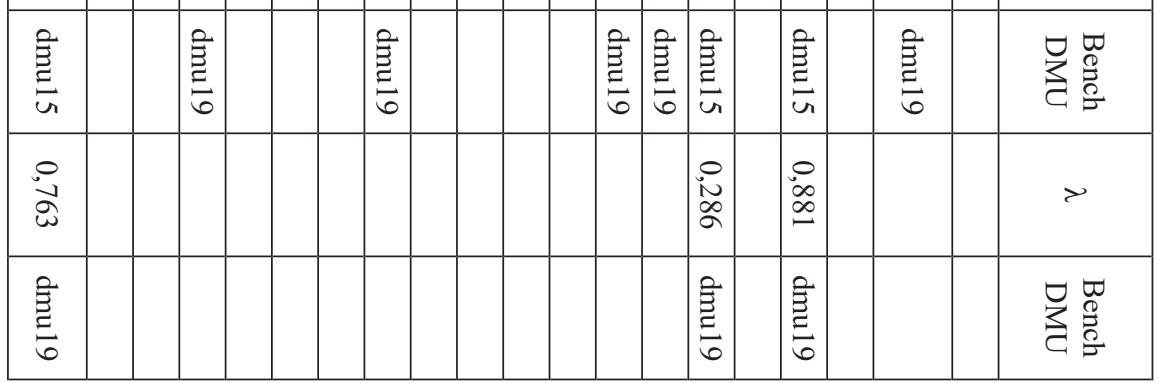


There are six countries that are efficient in 2008 under the CRS assumption for the overall process: Greece, Cyprus, Luxembourg, Malta, the Netherlands and Slovakia. From CEE countries only Slovakia is efficiency frontier. Other fourteen countries have scores of less than 1 , but greater than 0 and thus they are identified as inefficient. These countries can improve their efficiency, or reduce their inefficiencies proportionately, by reducing their inputs. Portugal obtained the worst result 0,4323 and could improve its efficiency by reducing R\&D expenditures up to $56,5 \%$. Romania $(0,95037)$, Ireland $(0,85464)$ and Bulgaria $(0,83439)$ are the closest to an efficiency frontier, and need accordingly $4,9 \%, 14,5 \%$ and $16,5 \%$ reduction in resources. Benchmarks (Bench DMU) are effective units. Ineffective units should follow the benchmarks DMUs innovation policy or organizational solutions in order to identify the best practices and their possible adaptation to improve their expenditure transformation processes in results. For instance, Benchmarks for Bulgaria is Cyprus and Slovakia. Bulgaria will attempt to become like Slovakia more than Cyprus as observed from respective lambda weights $\lambda=0,543$ and $\lambda=0,321$.

Under VRS assumption there are 12 countries that are efficient for the overall process: the Czech Republic, Ireland, Greece, Cyprus, Lithuania, Luxembourg, Hungary, Malta, the Netherlands, Romania, Slovakia and United Kingdom. From CEE region efficiency frontiers are the Czech Republic, Lithuania, Hungary, Romania and Slovakia. Similar to CRS Portugal is the least efficient. More countries are efficient under VRS assumption. Due to the definition, all relatively CRS efficient countries are scale efficient too. 


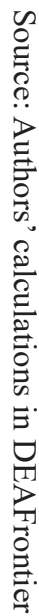

\begin{tabular}{|c|c|c|c|c|c|c|c|c|c|c|c|c|c|c|c|c|c|c|c|c|}
\hline$\widetilde{\sigma}$ & $\vec{\sigma}$ & $\vec{\infty}$ & 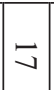 & $\vec{a}$ & $\vec{u}$ & $\vec{\Phi}$ & $\vec{\omega}$ & $\bar{N}$ & $=$ & $\overrightarrow{0}$ & 0 & $\infty$ & $\checkmark$ & $a$ & $u$ & $\Delta$ & $\omega$ & $N$ & -1 & Z \\
\hline$\underset{\stackrel{\Xi}{D}}{\stackrel{c}{D}}$ & 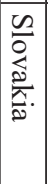 & 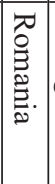 & 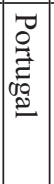 & 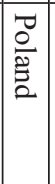 & 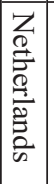 & $\begin{array}{l}3 \\
\stackrel{2}{2} \\
\stackrel{2}{2}\end{array}$ & 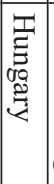 & 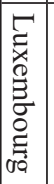 & | & $\underset{2}{2}$ & 辞 & $\overrightarrow{\overrightarrow{\vec{v}}}$ & 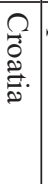 & 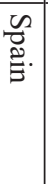 & $\begin{array}{l}\Omega \\
8 \\
\varnothing \\
\varnothing \\
\delta\end{array}$ & 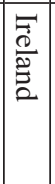 & 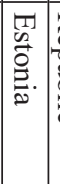 & 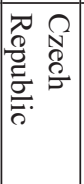 & 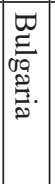 & 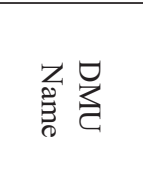 \\
\hline & : & : & $\mid \begin{array}{l}0 \\
\text { in } \\
0 \\
0\end{array}$ & $\begin{array}{l}0 \\
\stackrel{0}{*} \\
\vec{D}\end{array}$ & 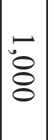 & : & 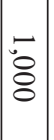 & 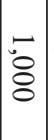 & $\mid$ & \begin{tabular}{l|l}
0 & \\
\multirow{y}{0}{} &
\end{tabular} & 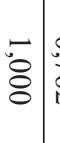 & $\begin{array}{l}0 \\
\stackrel{0}{N} \\
\stackrel{a}{ }\end{array}$ & : & $\begin{array}{l}\circ \\
\vdots \\
9 \\
\end{array}$ & : & 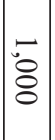 & 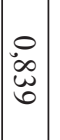 & ஜ & $\mid \begin{array}{l}0 \\
0 \\
0 \\
\dot{1} \\
\end{array}$ & 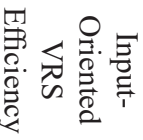 \\
\hline & 5 & $\div$ & $\begin{array}{l}0 \\
0 \\
1\end{array}$ & 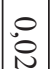 & 5 & $\div$ & 5 & $\div$ & $\div$ & : & 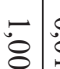 & : & م. & 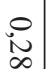 & 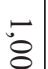 & 5 & $\stackrel{0}{N}$ & 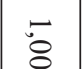 & $\mid \begin{array}{ll}0 \\
\dot{y} \\
0\end{array}$ & \\
\hline
\end{tabular}

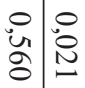

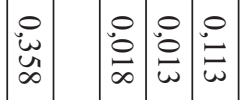

品

总导

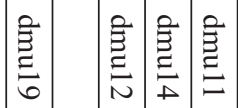

害

를

\begin{tabular}{ll}
$\bigcirc$ & 0 \\
\hdashline & $\infty$ \\
\cline { 1 - 1 } & $\sigma$
\end{tabular}

\begin{tabular}{llll}
0 & 0 & 0 \\
$\dot{w}$ & & 0 & 0 \\
+ & 0 & 0 \\
\hline
\end{tabular}

$\because 0$

$>$

䍚

总总尽

产

ํํㅇ

$\stackrel{0}{\circ}$

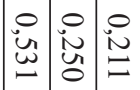

尽

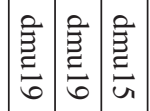

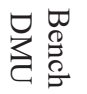


To expand the analysis, the author assessed the efficiency of spending on innovation for an additional seven years (2009-2015), for which similar procedure was carried out. The final results were presented and analyzed in the results and discussion section in tables 8,9 and 10 within the appendix. Diagnostic data of inputs and outputs for years 2009-2015 are presented in appendix as well.

\section{Results and discussion}

The conducted analysis allowed calculating efficiency indicators for $20 \mathrm{EU}$ economies. Ten of them are from CEE region: Bulgaria, the Czech Republic, Estonia, Croatia, Latvia, Lithuania, Hungary, Poland, Romania and Slovakia. Research period covered eight years from 2008 to 2015. R\&D expenditure efficiency is calculated by the CRS and VRS models. In addition, average efficiency indicator was calculated and change in indicator between 2015 and 2008. The average efficiency score is the arithmetic average of period efficiency scores during the eight years. Table 8 shows the final efficiency index for CRS and table 9 for VRS.

Based on table 10, Cyprus was identified the most efficient country, as it is efficient for each year under both CRS and VRS. According to VRS method other efficiency frontiers for period 2008-2015 are also Luxembourg, Malta, the Netherlands and Slovakia. Although Cyprus is not an innovative leader, the analysis proved that this economy is characterized by the most favorable ratio of expenditures to outputs. The second in ranking is Malta $(0.991)$. From CEE region third Romania $(0,976)$ and fourth Slovakia (0.971) are the closest to an efficiency frontier, and need accordingly $2,4 \%$ and $2,9 \%$ reduction in resources to become fully efficient. Latvia obtained the average efficiency index with value of 0.892 , which is above average for CEE region. Croatia, Bulgaria, Poland, Lithuania, the Czech Republic, Hungary and Estonia are below average for CEE countries. The worst efficiency index was obtained by Estonia. Position of Estonia may be surprising, because it is seen as one of the most innovative countries in the CEE region; however, it has the second highest average R\&D spending $1,67 \%$ of GDP. As demonstrated by quantitative research, huge innovation spending funds does not produce proportionally large results. In conclusion it is worth noting, that DEA methodology is calculating technical efficiency, which examines the degree to which R\&D expenditures have been transformed into potential of innovation.

Input indicator has significantly different values for the studied countries (from 0.46 to $1.86 \%$ GDP), and it has huge impact for the efficiency indicator. Spending on R\&D for studied countries is much below the EU28 average, which is approximately $2 \%$ of GDP. The highest spending during research period was noted in the Netherlands with average value $1.86 \%$ GDP, reaching 2,0\% of GDP in 2014-2015. Estonia and the 
Czech Republic are spending around 1,6\% of GDP on R\&D, and those are the highest values from CEE countries. The lowest R\&D expenditures were noted in Romania with average for period 2008-2016 0.46\% of GDP and Cyprus with average $0.46 \%$ of GDP. It is worth to emphasize that top four countries in the ranking are spending less than $0,75 \%$ of GDP on R\&D. Luxemburg and the Netherlands, ranked $5^{\text {th }}$ and $6^{\text {th }}$, are spending much more, respectively $1,42 \%$ and $1,86 \%$, which means that these countries had to achieve significantly better innovative outputs.

Outputs indicators are presented as comparable data, e.g. divided by million inhabitants or percentage. The Netherlands is achieving the best results for such output indicators as patent applications to the European Patent Office by priority year per mln inhabitants (PA), scientific and technical journal articles per mln inhabitants (ATJA), high-tech patent applications to the European Patent Office by priority year for mln inhabitants (PA-HT). Luxemburg has the biggest share of human resources in science and technology in active population (HRST), European Union trade mark applications per million population (EUTM) and employment in knowledge-intensive service sectors as \% of total employment (EHT-S). The highest share of employment in high- and medium-high technology manufacturing sectors (EHT-M) is in the Czech Republic. Also, Malta is leading in export of high-tech products (HTE). Lithuania has the highest share in graduates in tertiary education, in science, math., computing, engineering, manufacturing, construction (GTE). It is worth to underline that western countries, such as the Netherlands and Luxemburg, despite high R\&D spending are able to obtain very good innovative outputs. In terms of CEE countries, the leaders of R\&D spending - Estonia and the Czech Republic are achieving disproportionate outcomes. Hypothesis that increasing spending on innovations is not causing proportional effects has been confirmed for CEE region, but not for some western economies, which are spending on R\&D more effectively.

It is interesting to notice, that Croatia made the most significant improvement in efficiency indicator - according to CRS method from 0,440 in 2008 to 0,874 in 2015. During period 2008-2015 R\&D spending measured as GDP percentage did not change much, but in the same time all outputs indicators chosen for the DEA model increased. Portugal also improved innovation efficiency, without notable change in R\&D spending or even decrease. Slovakia, which is efficiency leader in years 2008-2012, lost its position due to increase in R\&D spending without proportional increase in output indicators.

Undoubtedly, innovation means different things depending on where a country stands in terms of development. Overall, significant national and regional disparities exist in the innovative environment in Europe. Northern and northwestern European countries are performing strongly compared with a lagging southern Europe and Central and Eastern Europe. According to the European Innovation Index (European Commission, 2017) The Netherlands, The United Kingdom are innovative leaders. Romania and Bulgaria are classified as modest 
innovators, Luxemburg as strong innovators, while all other studied countries are classified as moderate innovators. Innovation leaders can take advantage related to commercialization of emerging technologies and spreading innovation across regional or global markets. Countries less technologically advanced should focus on catch-up strategies. The adoption of innovative technologies and creating favourable conditions for the innovation development should be key area of innovation strategies. Competitiveness and innovativeness divide will require differentiated strategies, that take national and regional characteristics into account. Investments in knowledge-generating assets will convert into important drivers for future productivity growth.

\section{Conclusions}

Research on efficiency of R\&D spending expends current scientific knowledge. The results of the research confirmed hypothesis, that higher R\&D spending is not causing proportional innovative effects for CEE region, but not for some western economies, which are spending on R\&D more effectively. DEA provides results for technical efficiency, which examines how public and private expenditure have been converted into the effects. The efficiency indicator informs about the efficiency of the use of funds. Countries with the highest R\&D spending do not necessarily achieve the best innovative results. It is worth to mention, that currently international organizations are working on more sufficient innovation input statistics such as stock of current knowledge, number of innovative enterprises, R\&D expenditures, human resources and research infrastructures, which can provide more actual picture of efficiency. Also, innovative outcomes are hard to present in statistics. Launching a patent or new technology will cause additional economic profits in the future. However, they are hard to estimate and time delay should be also considering. Including such inputs and outputs into DEA methodology would provide better overview of efficiency of innovative actions in the economy. However, nowadays such statistics are not yet fully available. Author in this research was limited by available data, as countries comparative studies require comparable and uniform statistics. Moreover, analysis of longer period could bring more general conclusion and recommendations for innovative policies. Further research can be conducted for EU regions, as in each country there are huge differences between regions in terms of innovation capabilities. Even the $\mathrm{EU}$ is recently focusing more on regions, than countries. Competitiveness of EU is assessed at regional level in European Regional Competitiveness Index, where one of three dimensions is innovation. Area of research in this article covered only innovation factor. Analysing efficiency of other types of country spending may acquire a general efficiency indicator, which will allow classifying countries according to their efficiency level. Such analysis would answer the question on which state policy is the most effectively using available resources. Finally, the 
results of this study can be used by policymakers working on innovation policies in CEE countries. Research confirms that CEE countries are not able to achieve innovation outputs proportional to the R\&D spending. For those countries where innovative capacities are still limited more reasonable seems to be step by step policy. Gradual increase in investment in innovation may produce better conditions for innovation-driven growth.

\section{References}

Anvret, M., Granieri, M., Renda, A. (2010) "A New Approach to Innovation Policy in the European Union. Innovation Policy: Boosting EU Competitiveness in a Global Economy", CEPS Task Force Report, [Online] Available at: http://aei. pitt.edu/14482/, [Accessed 20 March 2018].

Carlin, W., Soskice D. (2006) "Macroeconomics: Imperfections, Institutions and Policies", Oxford: Oxford University Press.

Cook, W.D., Tone, K., Zhu, J. (2014) "Data envelopment analysis: Prior to choosing a model", Omega, 44, pp. 1-4, doi: 10.1016/j.omega.2013.09.004.

Charnes, A., Cooper, W.W., and Rhodes, E. (1978) "Measuring the efficiency of decision making units", European Journal of Operational Research, Vol. 2, No. 6, pp. 429-444, doi: 10.1016/0377-2217(78)90138-8.

Chen, C. P., Hu, J. L., Yang, C. H. (2013) "Produce patents or journal articles? A cross-country comparison of R\&D productivity change", Scientometrics, Vol. 94, No. 3, pp. 833-849, doi: 10.1007/s11192-012-0811-9.

Cooper J.O, Heron T.E, Heward W.L. (2007) “Applied behavior analysis” (2nd ed.) Upper Saddle River, NJ: Pearson.

Cruz-Cázares, C., Bayona-Sáez C., García-Marco T. (2013) "You can’t manage right what you can't measure well: Technological innovation efficiency", Research Policy, Vol. 42, No. 6, pp. 1239-1250, doi: 10.1016/j.respol.2013. 03.012 .

DeVolpi, A. (1991) "Understanding correlation coefficients in treaty verification", doi: $10.2172 / 10167858$.

European Commission (2010) "Europe 2020. A strategy for smart, sustainable and inclusive growth", [Online] Available at: http://ec.europa.eu/eu2020/pdf/ COMPLET\%20EN\%20BARROSO\%20\%20\%20007\%20-\%20Europe\%20 2020\%20-\%20EN\%20version.pdf, [Accessed 23 Feb 2018].

European Commission (2011), "Europe 2020 Flagship Initiative Innovation Union", [Online] Available at: https://ec.europa.eu/research/innovation-union/pdf/ innovation-union-communication-brochure_en.pdf, [Accessed 26 March 2018].

European Commission (2013a) "Europe 2020: Europe's growth strategy: growing to a sustainable and job-rich future", [Online] Available at: https://publications. 
europa.eu/en/publication-detail/-/publication/cf3b4a6f-6d45-4335-8624ac12e44ef020, [Accessed 23 Feb 2018].

European Commission (2013b), "Measuring Innovation Output in Europe: Towards a New Indicator", [Online] Available at: https://ec.europa.eu/research/ press/2013/pdf/indicator_of_innovation_output.pdf, [Accessed 23 Feb 2018].

European Commission (2017), "European Innovation Scoreboard", [Online] Available at: http://ec.europa.eu/growth/industry/innovation/facts-figures/scoreboards_en, [Accessed 23 Feb 2018].

Eurostat Data site. Available: http://ec.europa.eu/eurostat/data/database [Accessed: 15 March 2018].

Farrell M.J. (1957) "The measurement of productive efficiency", Journal of the Royal Statistical Society, Vol. 120, No. 3, pp. 253-290, doi: 10.2307/2343100.

Fagerberg, J., Srholec, M. (2008) "National innovation systems, capabilities and economic development", Research Policy, Vol. 37, No. 9, pp. 1417-1435. doi: 10.1016/j.respol.2008.06.003.

Gartner, W.B., Drucker, P.F. (1987) "Innovation and Entrepreneurship" The Academy of Management Review, Vol. 12, No. 1, p. 172, doi: 10.2307/258006.

Gros D., Roth F. (2012) “The Europe 2020 Strategy can it maintain the eu's competitiveness in the world?", Brussel: Centre for European Policy Studies.

Guan, J., Chen K. H. (2012) "Modeling the relative efficiency of national innovation systems", Research Policy, Vol. 41, No. 1, pp. 102-115, doi: 10.1016/j.respol. 2011.07.001.

Guan, J., Zuo, K. (2014) "A cross-country comparison of innovation efficiency", Scientometrics, Vol. 100, No. 2, pp. 541-575, doi: 10.1007/s11192-014-1288-5.

Hashimotoa, A., Haneda, S. (2008) "Measuring the change in R\&D efficiency of the Japanese pharmaceutical industry", Research Policy, Vol. 37, No. 10, pp. 1829-1836, doi: 10.1016/j.respol.2008.08.004.

He, B., Wang, J., Wang, J., Wang, K. (2018) “The Impact of Government Competition on Regional R\&D Efficiency: Does Legal Environment Matter in China's Innovation System?", Sustainability, Vol. 10, No. 12, 4401, doi: 10.1016/0377-2217(78)90138-8.

Hirooka, M. (2006) "Innovation Dynamism and Economic Growth", Cheltenham: Edward Elgar Publishing, doi: 10.4337/9781845428860.

Janger, J. et al. (2017) "The EU 2020 innovation indicator: A step forward in measuring innovation outputs and outcomes?", Research Policy, Vol. 46, No.1, pp.30-42. doi: 10.1016/j.respol.2016.10.001.

Jenkins L., Anderson M. (2003) "A multivariate statistical approach to reducing the number of variables in data envelopment analysis", European Journal of Operational Research, Vol. 147, No. 1, pp. 51-61, doi: 10.1016/S0377-2217(02) 00243-6. 
Liu, J.; Lu, K.; Cheng, S. (2018) "International R\&D Spillovers and Innovation Efficiency”, Sustainability, Vol. 10, No. 11, 3974. doi: 10.3390/su10113974.

Molinero, C.M., Woracker, D. (1996) "Data Envelopment Analysis: OR Insight, Vol. 9, No. 4, pp. 22-28, doi: http://dx.doi.org/10.1057/ori.1996.21.

Pelkmans, J., Renda, A. (2014) "Does EU Regulation Hinder or Stimulate Innovation?", CEPS Special Report No. 96. [Online] Available at: https://ssrn. com/abstract $=2528409$ [Accessed 27 March 2018].

Romer, P.M. (1986) "Increasing Returns and Long Run Growth", Journal of Political Economy, Vol. 98, pp. 71-102, doi: 10.1086/261420.

Sharma, S., Thomas, V.J. (2008) "Inter-country R\&D efficiency analysis: An application of data envelopment analysis", Scientometrics, Springer, Vol. 76, No. 3, pp. 483-501, doi: 10.1007/s11192-007-1896-4.

Solow, R. (1956) "A Contribution to the Theory of Economic Growth", The Quarterly Journal of Economics, Vol. 70, No. 1, pp. 65-94, doi: 10.2307/ 1884513.

Schumpeter, J., Backhaus, U. (2003) "The Theory of Economic Development”, The European Heritage in Economics and the Social Sciences, Vol. 1, pp. 61-116. doi: 10.1007/0-306-48082-4_3.

Thanassoulis, E. (2001) "Using Data Envelopment Analysis in Practice. Introduction to the Theory and Application of Data Envelopment Analysis", pp. 89-121. doi: 10.1007/978-1-4615-1407-7_5.

Thanassoulis E. (2003) "Introduction to the Theory and Application of Data Envelopment Analysis", Norwell: Kluwer Academic Publishers, doi: 10.1007/ 978-1-4615-1407-7.

Tilford, S., Whyte P. (2010) “The Lisbon Scorecard X: The road to 2020", Centre for European Reform, London, [Online] Available at: http://www.cer.eu/sites/ default/files/publications/attachments/pdf/2011/rp_967-251.pdf [Accessed 23 Feb 2018].

Taylor, M.S., Grossman, G.M., Helpman, E. (1993) "Innovation and Growth in the Global Economy", Economica, Vol. 60, No. 239, p. 373, doi: 10.2307/2554862.

World Bank Data site. Available: https://data.worldbank.org/ [Accessed: 15 March 2018].

Zabala-Iturriagagoitia, J.M., (2008), "Benchmarking Regional Innovation Systems: the relevance of efficiency towards their performance", doi: /10.4995/thesis/ $10251 / 3787$.

Zuo, K., Guan J. (2017) "Measuring the R\&D efficiency of regions by a parallel DEA game model", Scientometrics, Springer, Vol. 112, No. 1, pp. 175-194, doi: 10.1007/s11192-017-2380-4.

Wober K. W. (2007) "Data Envelopment Analysis", Journal of Travel \& Tourism Marketing, Vol. 21, No. 4, pp. 91-108, doi: 10.1300/j073v21n04_07. 


\title{
Efikasnost ulaganja u investicije u zemljama Srednje i Istočne Europe
}

\author{
Pawel Dobrzanski ${ }^{1}$
}

\begin{abstract}
Sažetak
Svrha ove studije je provjeriti koristi li se novac na istraživanje i razvoj efikasnosti u zemljama srednje $i$ istočne Europe. Danas je inovativnost jedan od najvažnijih čimbenika ubrzanja gospodarskog rasta. Povećanje inovativnosti osobito je važno za zemlje u razvoju, gdje kreatori politike provode različite inovacijske strategije. Strategija Europa 2020 postavila je cilj 3\% BDP-a za izdatke za istraživanje $i$ razvoj. Mnoge studije naglašavaju značajan učinak povećanja ulaganja u istraživanje i razvoj na gospodarski rast, ali aspekt efikasnosti nije obuhvaćen u literaturi. Ovaj rad temelji se na kritičkom pregledu relevantne literature o ovom predmetu $i$ vlastitim empirijskim istraživanjima. Izvor statističkih podataka korištenih u ovoj studiji je relevantna međunarodna statistika. Izračuni su izvedeni korištenjem DEA metodologije. DEA metodologija omogućuje procjenu efikasnosti input-outputa. Pokazatelj inputa je godišnja javna $i$ privatna potrošnja na istraživanje i razvoj (kao\% BDP-a). Postoji devet pokazatelja outputa/rezultata koji predstavljaju dostupne inovativne statistike o broju patenata, visokotehnološkoj proizvodnji itd. Broj varijabli se smanjuje za svako razdoblje korištenjem analize korelacijskog koeficijenta, što je omogućilo identificiranje značajnih varijabli s najmanjim gubitkom podataka. Efikasnost se izračunava kao omjer ponderiranog zbroja outputa prema ponderiranom zbroju inputa odnosno ulaznih vrijednosti. Izračun je rađen korištenjem Excel proračunske tablice i DEAFrontier-a. U radu se daje opći pregled razine inovacija u zemljama srednje i istočne Europe u usporedbi s ostalim članicama EU-a, koji troše manje od 2\% BDP-a na istraživanje i razvoj. Analiza pokazuje da među zemljama srednje $i$ istočne Europe najbliže granici efikasnosti su Rumunjska i Slovačka. Hipoteza da povećanje potrošnje na inovacije ne uzrokuje proporcionalne učinke potvrđena je za regiju Srednje i Istočne Europe, ali ne i za zapadne ekonomije koje troše na istraživanje i razvoj efikasnije. Glavni zaključak istraživanja je da se potrošnja na inovacije treba postupno povećavati u cilju postizanja optimalnih rezultata. Ovo istraživanje može doprinijeti raspravi o kreiranju politike inovacija koju kreatori politike mogu koristiti za razvoj nacionalnih strategija inovacija.
\end{abstract}

Ključne riječi: inovacija, DEA metodologija, relativna efikasnost, investicije

JEL klasifikacija: H50, O30, O38, O57, R15

1 Docent, Wroclaw University of Economics, Faculty of Economics, 118/120 Komandorska St, 53-345 Wroclaw, Poljska. Znanstveni interes: međunarodna ekonomija. Tel.:+48601594346, E-mail:pawel.dobrzanski@ue.wroc.pl. 


\section{Appendices}

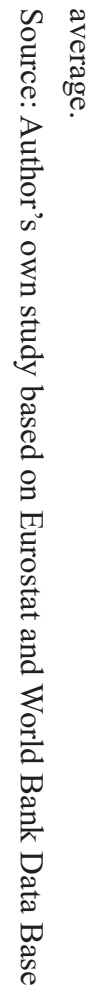

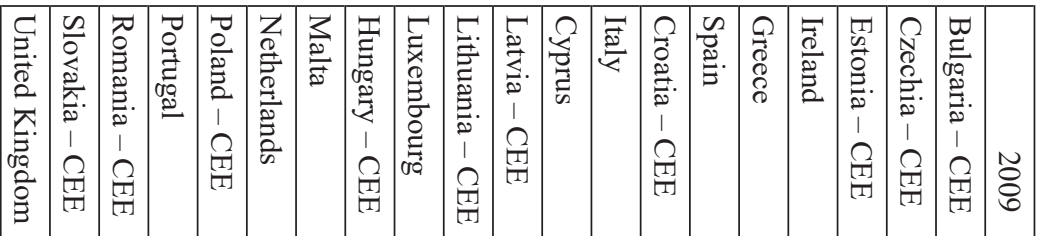

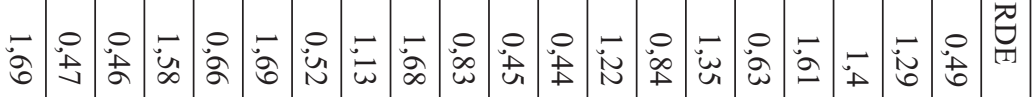

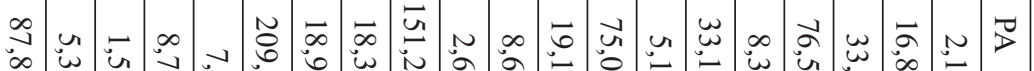

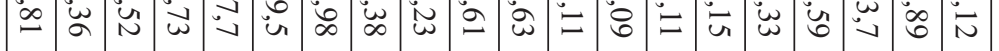

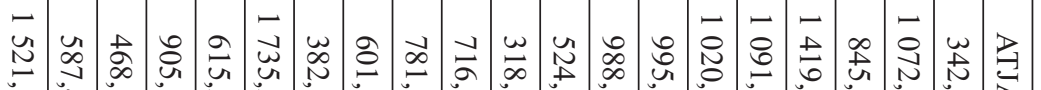
苻古

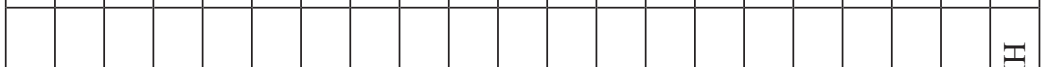

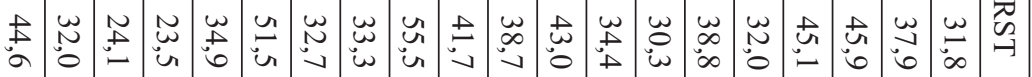

$-000 .-0.0$

¿.

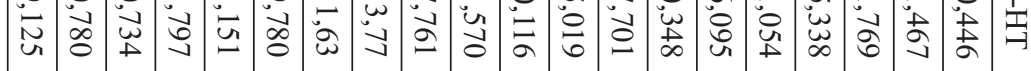

है:

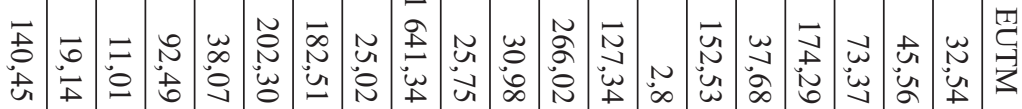

峁

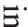

ह

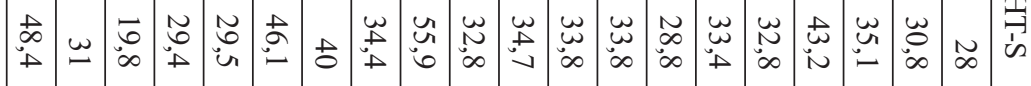

$\stackrel{9}{\circ}$

?.

苗.

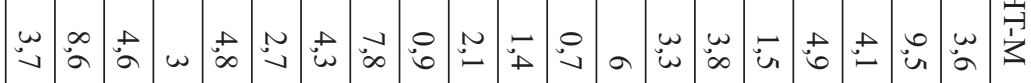

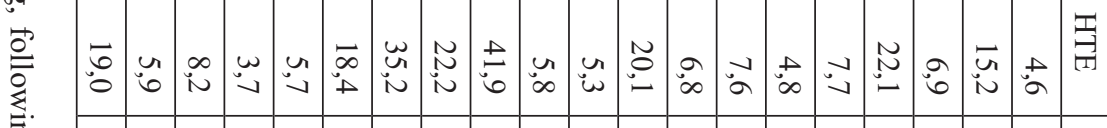
言

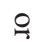
蛋.

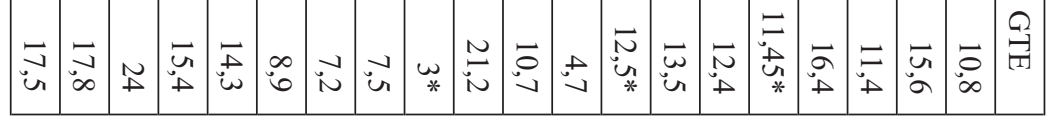




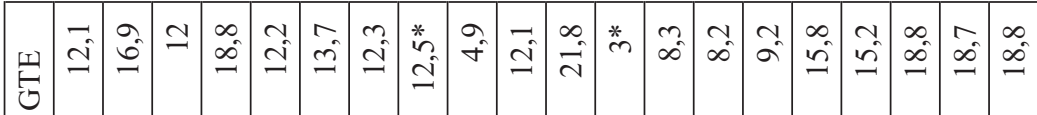

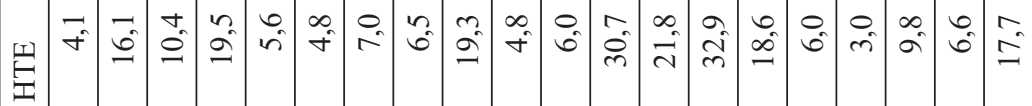

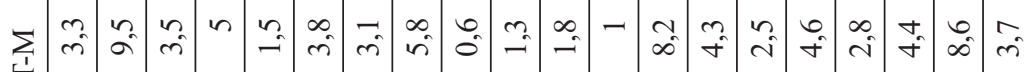

空

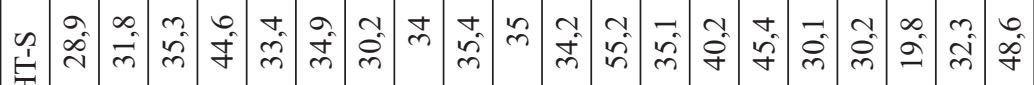

空

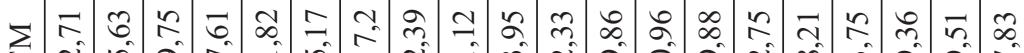

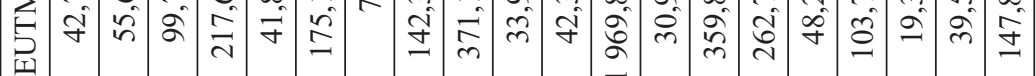

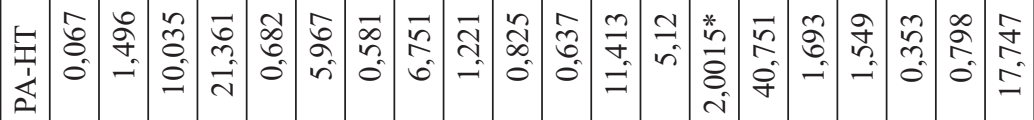

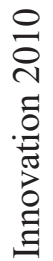

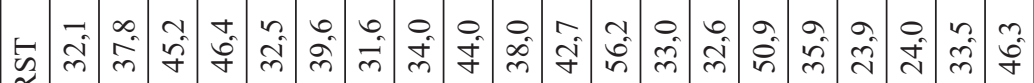

步

«

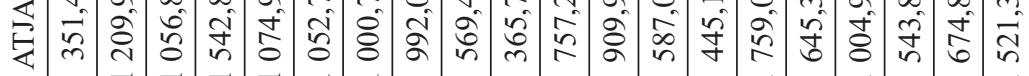

泀

ริ

ङ

II $\simeq$

宽

$\cdot \frac{0}{0}$

कू⿱

ค.

ij

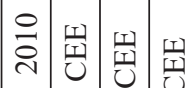

띤

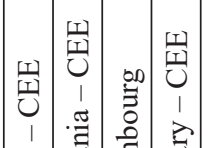

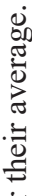

ธั

言

.

这

물

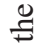

$\exists$

$\stackrel{\frac{2}{3}}{\frac{\pi}{2}}$

ฮั

$\frac{\sqrt{0}}{3}$

$\ll$ 


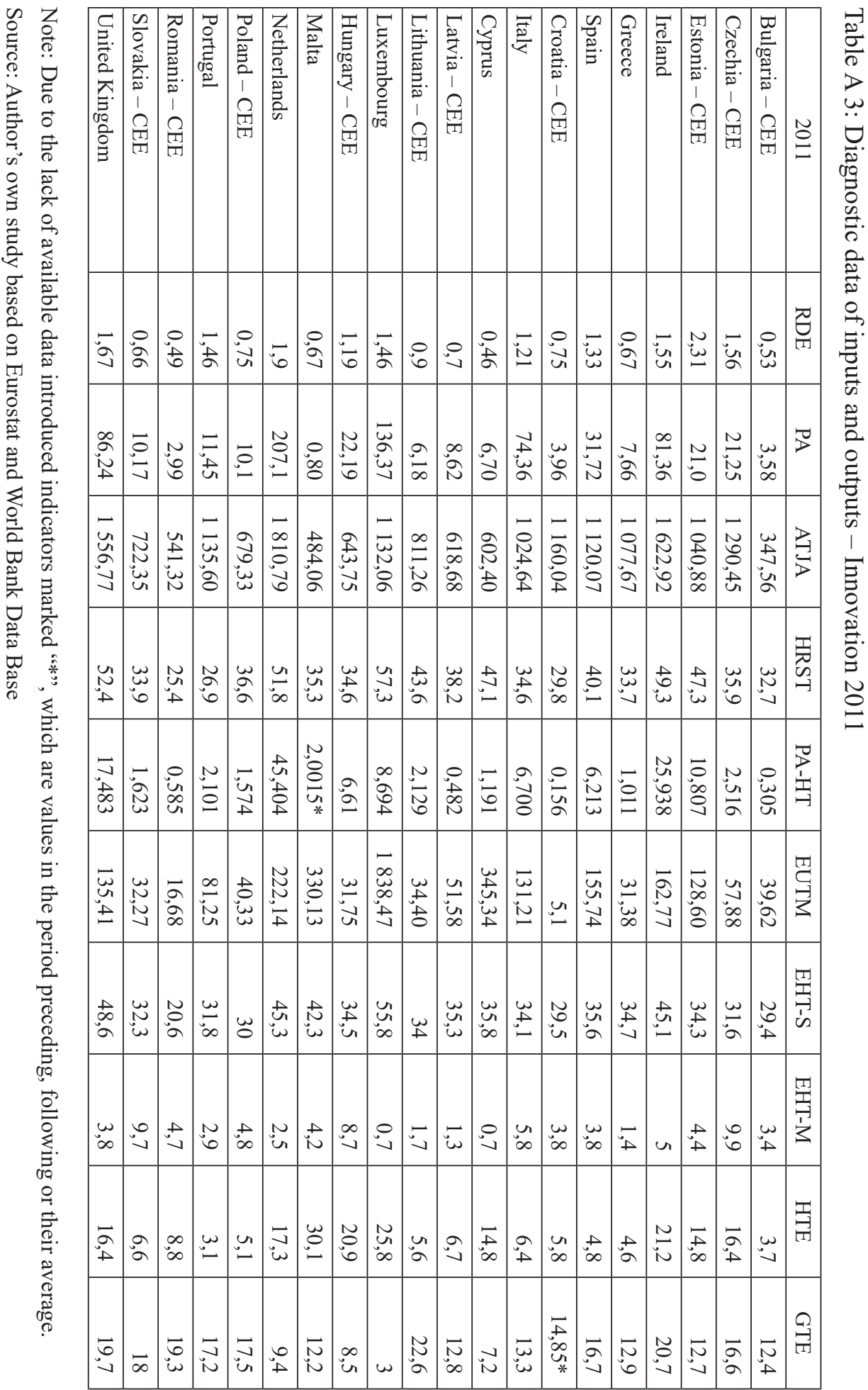




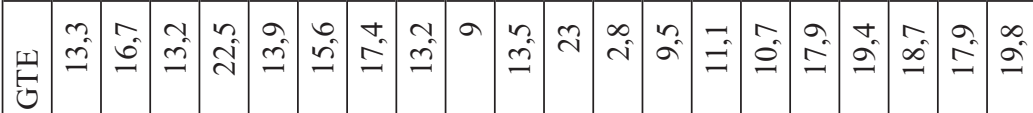

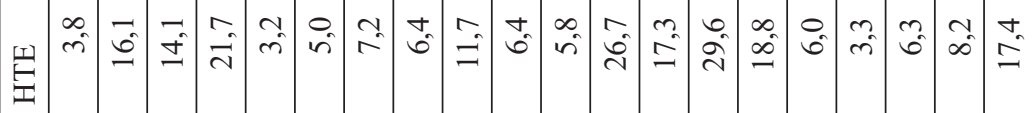

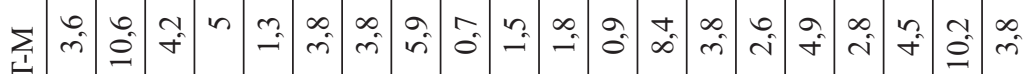

空

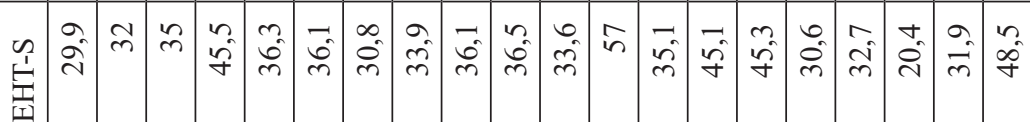

$\Sigma$ ป

它

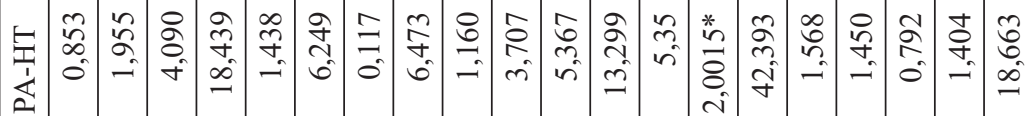

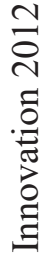

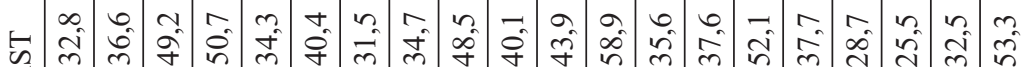

岂

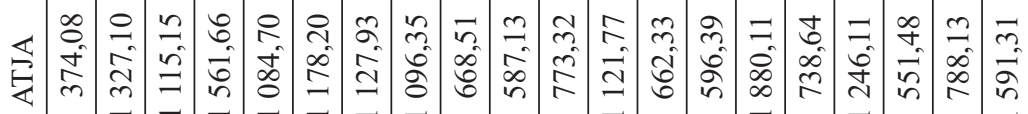

节

ชู

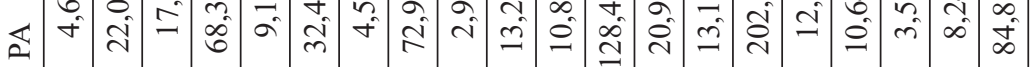

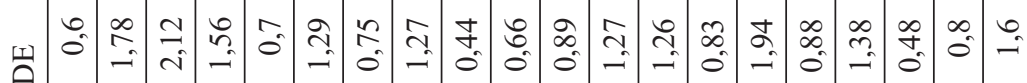

حิ

宽

$\cdot \stackrel{0}{*}$

कृ

$\stackrel{\frac{\pi}{a}}{\frac{\pi}{2}}$

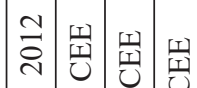

니

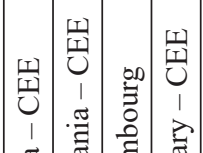

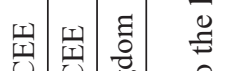

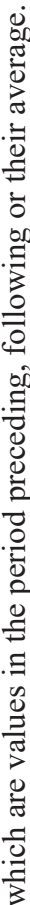

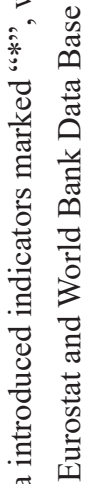

蒈

용

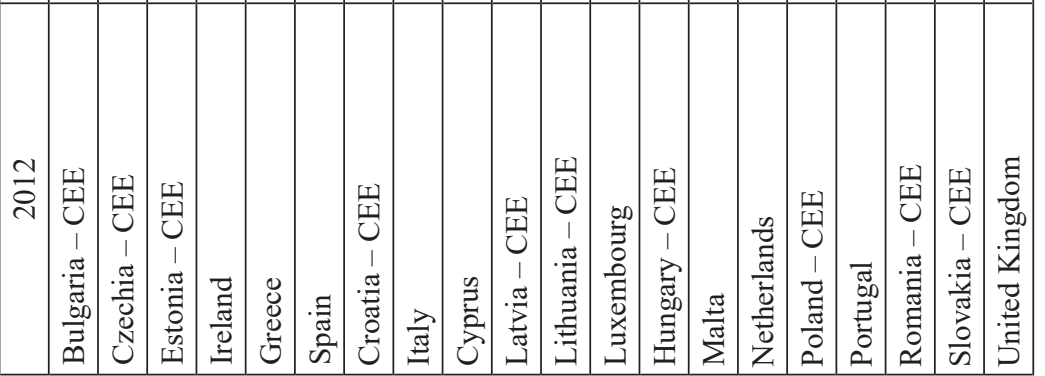




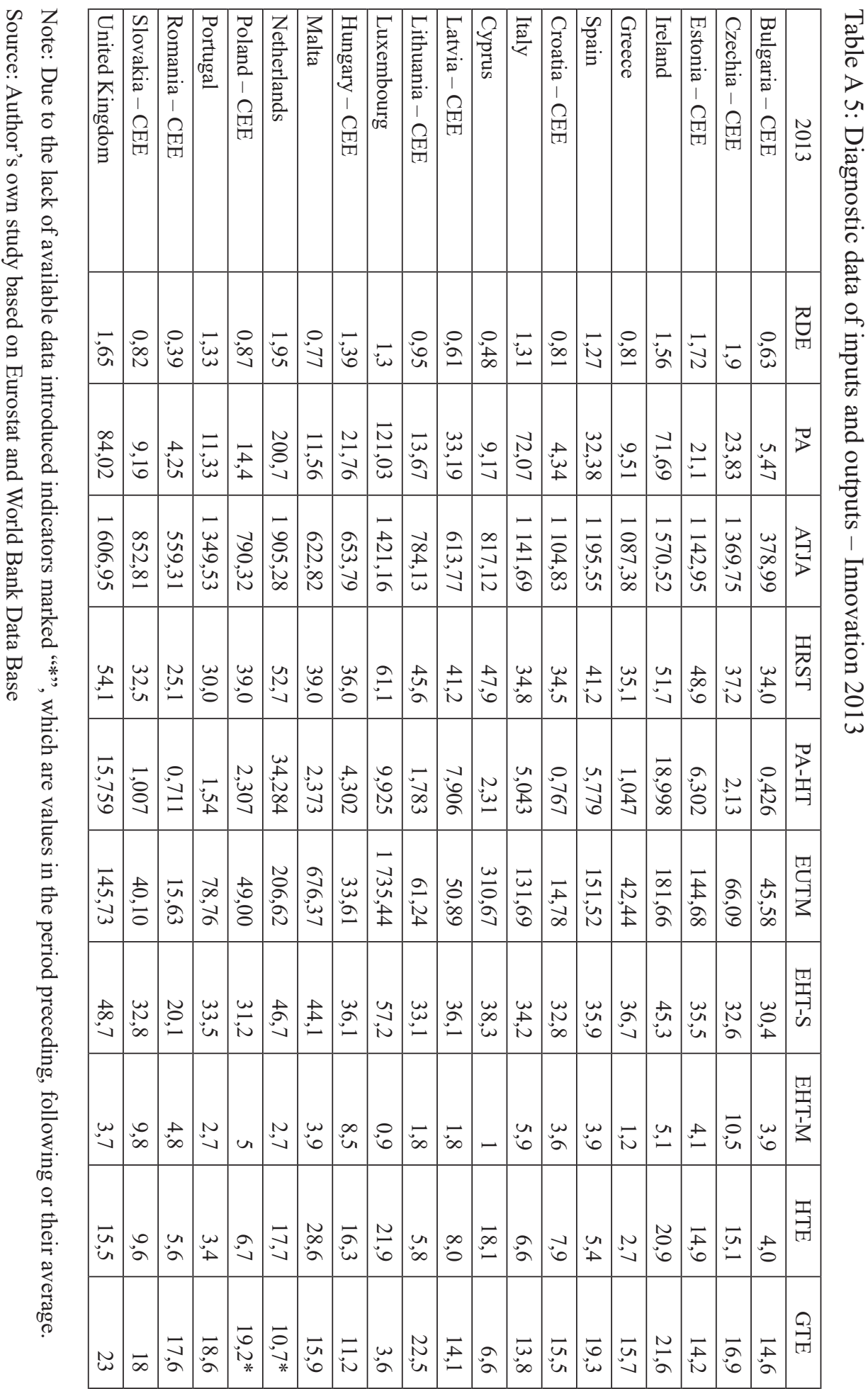




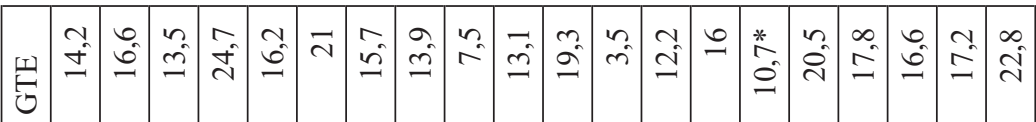

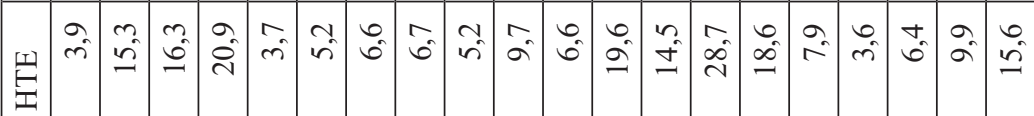

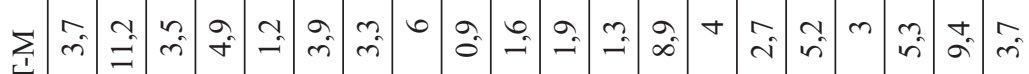

点

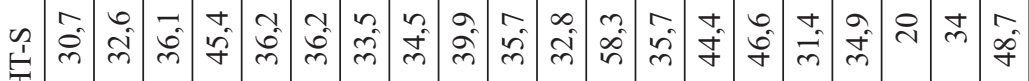

垔

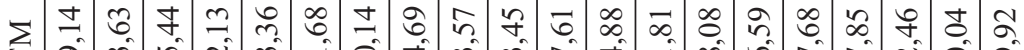

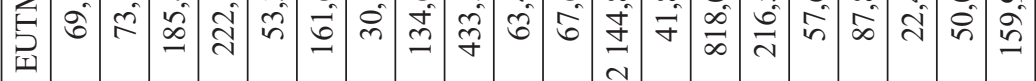

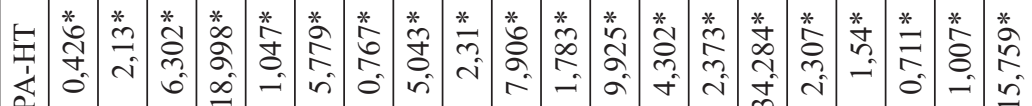

龒

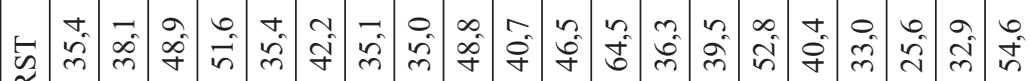

寻

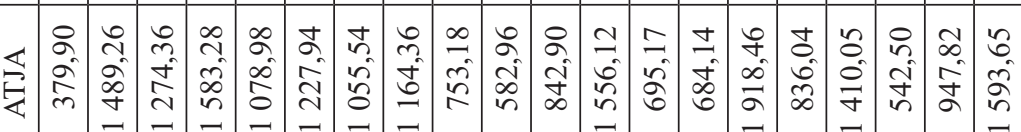

节

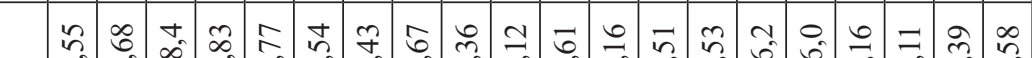

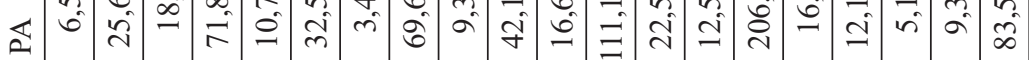

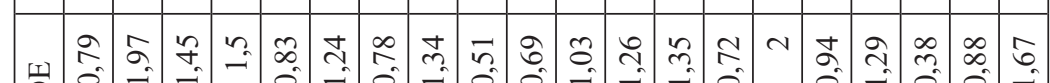

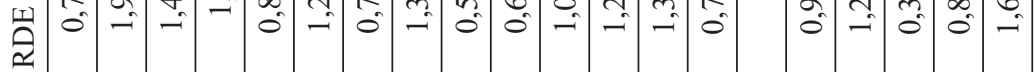

宽

$\cdot \frac{0}{0}$

ह10

$\stackrel{\frac{\pi}{0}}{\circ}$

6.

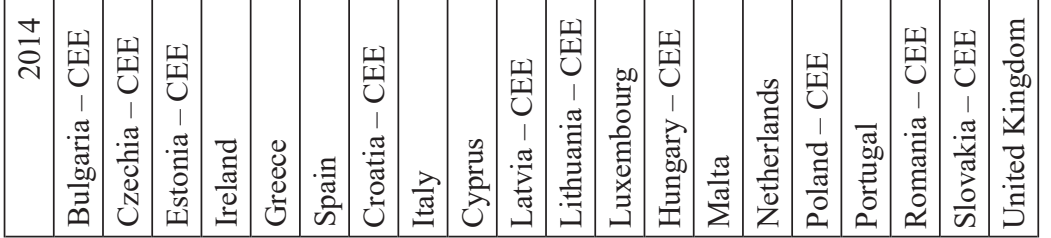

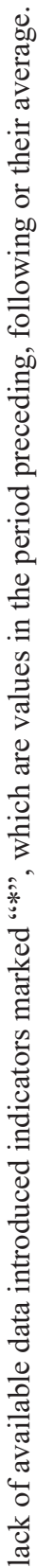

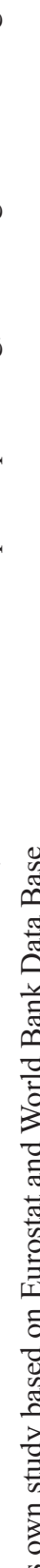

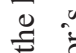

옹

$\stackrel{2}{2}$

$\frac{0}{0}$

훙 


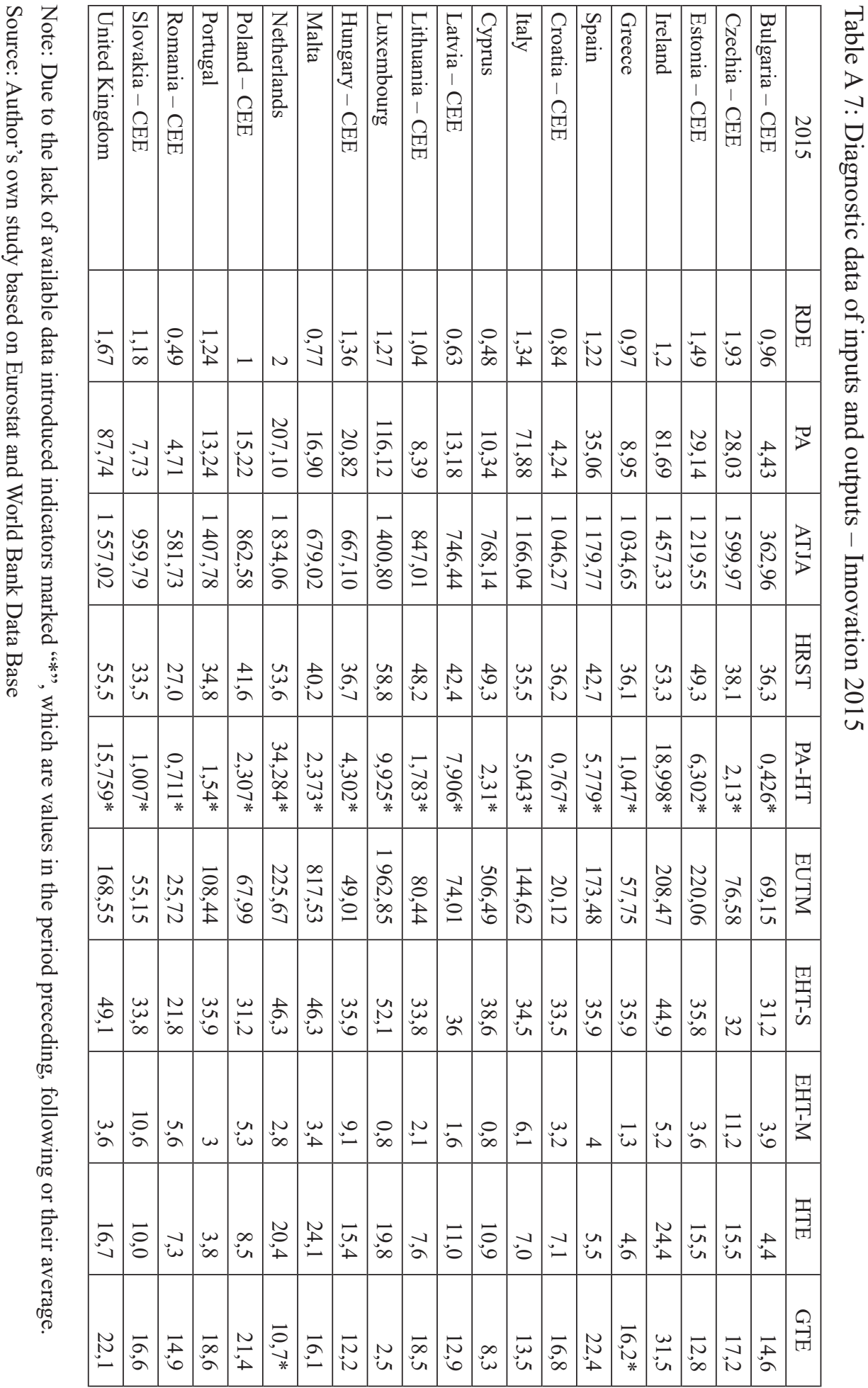




\begin{tabular}{|c|c|c|c|c|c|c|c|c|c|c|c|c|c|c|c|c|c|c|c|c|c|c|}
\hline 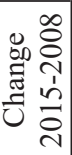 & $\begin{array}{l}\overrightarrow{\widehat{N}} \\
\hat{2} \\
\hat{1}\end{array}$ & $\begin{array}{l}n \\
\tilde{O} \\
\tilde{0}\end{array} \mid$ & $\left|\begin{array}{c}\infty \\
\tilde{0} \\
0 \\
1 \\
1\end{array}\right|$ & $\left|\begin{array}{l}\partial \\
\dot{J} \\
0\end{array}\right|$ & $\begin{array}{l}\stackrel{n}{ \pm} \\
\tilde{0}\end{array}$ & 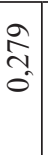 & \begin{tabular}{c}
$\stackrel{+}{f}$ \\
\multirow{f}{*}{} \\
0
\end{tabular} & 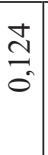 & $\begin{array}{l}8 \\
\vdots \\
0\end{array}$ & $\begin{array}{l}n \\
\tilde{n} \\
\tilde{0}\end{array}$ & $\begin{array}{c}\dot{0} \\
0 \\
0 \\
i\end{array}$ & 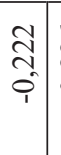 & 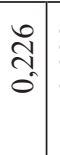 & $\mid \begin{array}{l}8 \\
8 \\
0 \\
0\end{array}$ & $\begin{array}{l}8 \\
8 \\
0 \\
0\end{array}$ & $\left|\begin{array}{l}2 \\
01 \\
0 \\
0 \\
1\end{array}\right|$ & $\left|\begin{array}{l}\infty \\
0 \\
2 \\
0\end{array}\right|$ & $\left|\begin{array}{l}0 \\
0 \\
0 \\
0\end{array}\right|$ & 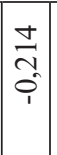 & $\begin{array}{l}n \\
\tilde{\delta} \\
0 \\
0\end{array}$ & $\begin{array}{l}\vec{n} \\
\tilde{0} \\
0\end{array}$ & $\left|\begin{array}{l}0 \\
0 \\
0 \\
0\end{array}\right|$ \\
\hline 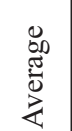 & $\left|\begin{array}{c}0 \\
n \\
0 \\
0\end{array}\right|$ & $\left|\begin{array}{l}0 \\
0 \\
2 \\
0\end{array}\right|$ & $\mid$\begin{tabular}{c}
0 \\
$\infty$ \\
\multirow{1}{\alpha}{} \\
0
\end{tabular} & $\left|\begin{array}{l}2 \\
\infty \\
2 \\
0\end{array}\right|$ & $\begin{array}{c}\underset{D}{D} \\
\hat{0}\end{array}$ & $\begin{array}{l}0 \\
⿱ \\
2 \\
2 \\
0\end{array}$ & $\begin{array}{c}\tilde{n} \\
\stackrel{n}{0}\end{array}$ & $\begin{array}{l}\overrightarrow{\widetilde{b}} \\
0 \\
0\end{array}$ & $\begin{array}{l}8 \\
\stackrel{-}{0}\end{array}$ & $\begin{array}{l}n \\
\infty \\
\infty \\
0\end{array}$ & $\begin{array}{l}2 \\
6 \\
0 \\
0\end{array}$ & 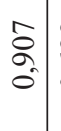 & $\begin{array}{l}\tilde{Z} \\
\text { ర్ } \\
0\end{array}$ & $\left|\begin{array}{l}\sqrt{0} \\
\sigma \\
0 \\
0\end{array}\right|$ & 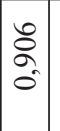 & $\left|\begin{array}{l}m \\
\hat{2} \\
0\end{array}\right|$ & $\left|\begin{array}{l}n \\
\infty \\
+ \\
\multirow{0}{*}{}\end{array}\right|$ & $\left|\begin{array}{l}n \\
\tilde{n} \\
\sigma\end{array}\right|$ & 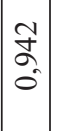 & $\begin{array}{l}0 \\
\& \\
0 \\
0 \\
0\end{array}$ & $\frac{\grave{N}}{\stackrel{0}{\circ}}$ & $\mid \begin{array}{c}n \\
\tilde{0} \\
0\end{array}$ \\
\hline$\stackrel{n}{\circ}$ & $\left|\begin{array}{l}2 \\
0 \\
n \\
0 \\
0\end{array}\right|$ & $\begin{array}{l}0 \\
\tilde{D} \\
0\end{array} \mid$ & $\mid \begin{array}{c}\infty \\
\infty \\
\hat{n} \\
0 \\
0\end{array}$ & $\left|\begin{array}{l}8 \\
8 \\
-1\end{array}\right|$ & $\frac{\mathfrak{m}}{\stackrel{+}{\sigma}}$ & $\begin{array}{c}\stackrel{0}{r} \\
\frac{0}{0}\end{array}$ & $\begin{array}{c} \pm \\
\Delta \\
0 \\
0\end{array}$ & $\begin{array}{l}8 \\
\mathbb{0} \\
0 \\
0\end{array}$ & $\begin{array}{l}8 \\
8 \\
-1\end{array}$ & $\underset{8}{8}$ & $\begin{array}{l}-7 \\
6 \\
0\end{array}$ & $\begin{array}{l}\infty \\
⿱ 乛 \\
\vdots \\
0\end{array}$ & $\begin{array}{l}\infty \\
\infty \\
0 \\
0 \\
0\end{array}$ & $\begin{array}{l}8 \\
8 \\
-1\end{array}$ & $\begin{array}{l}8 \\
8 \\
-1\end{array}$ & $\left|\begin{array}{l}m \\
\tilde{n} \\
\sigma\end{array}\right|$ & 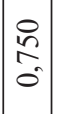 & $\begin{array}{l}8 \\
8 \\
-\end{array}$ & $\left|\begin{array}{l}0 \\
\infty \\
0 \\
0\end{array}\right|$ & $\underset{0}{\stackrel{\nabla}{*}}$ & $\begin{array}{c}\stackrel{+}{n} \\
\hat{0}\end{array}$ & $\mid \begin{array}{l}2 \\
\hat{2} \\
0\end{array}$ \\
\hline$\stackrel{+}{\stackrel{\sim}{\sim}}$ & $\mid$\begin{tabular}{c}
0 \\
\multirow{f}{*}{} \\
0
\end{tabular} & $\left|\begin{array}{l}2 \\
n \\
2 \\
0\end{array}\right|$ & \begin{tabular}{|c|}
$\infty$ \\
$\infty$ \\
0 \\
0 \\
0
\end{tabular} & $\mid \begin{array}{l}0 \\
8 \\
0 \\
-1\end{array}$ & $\begin{array}{l}\infty \\
\infty \\
\infty \\
0\end{array}$ & $\begin{array}{l}0 \\
\stackrel{2}{0} \\
0\end{array}$ & $\begin{array}{l}\stackrel{J}{\sigma} \\
\text { م. }\end{array}$ & $\begin{array}{l}\mathcal{I} \\
\mathcal{J} \\
0 \\
0\end{array}$ & $\begin{array}{l}8 \\
8 \\
-\end{array}$ & $\begin{array}{l}2 \\
\stackrel{\alpha}{ } \\
\hat{0}\end{array}$ & $\begin{array}{l}\hat{\sigma} \\
n \\
0\end{array}$ & $\begin{array}{l}8 \\
8 \\
- \\
-1\end{array}$ & $\begin{array}{l}\vec{\sigma} \\
0 \\
0\end{array}$ & $\begin{array}{l}8 \\
8 \\
-1\end{array}$ & 8 & $\left|\begin{array}{c}\infty \\
\widehat{6} \\
0 \\
0\end{array}\right|$ & 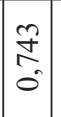 & $\begin{array}{l}8 \\
8 \\
- \\
-\end{array}$ & $\frac{N}{\stackrel{N}{0}}$ & $\begin{array}{c}\widehat{A} \\
\infty \\
0 \\
0\end{array}$ & $\frac{\pi}{\widehat{*}}$ & $\mid \begin{array}{l}8 \\
\vdots \\
\infty \\
0\end{array}$ \\
\hline & $\left|\begin{array}{l}J \\
0 \\
0\end{array}\right|$ & $\left|\begin{array}{l}0 \\
n \\
n \\
0\end{array}\right|$ & $\begin{array}{c}\mathbb{N} \\
\hat{n} \\
0\end{array}$ & $\begin{array}{l}\tilde{J} \\
0 \\
0\end{array}$ & $\begin{array}{l}n \\
\infty \\
0 \\
0 \\
0\end{array}$ & \begin{tabular}{l}
$n$ \\
\multirow{2}{*}{} \\
$\tilde{n}$
\end{tabular} & 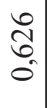 & $\begin{array}{c}\vec{\Xi} \\
\infty \\
0 \\
0\end{array}$ & $\underset{8}{8}$ & $\underset{-}{\stackrel{8}{\circ}}$ & $\begin{array}{l}\overline{6} \\
0 \\
0\end{array}$ & $\stackrel{8}{8}$ & $\begin{array}{l}\text { Oे } \\
0 \\
0\end{array}$ & 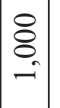 & $\stackrel{8}{8}$ & $\left|\begin{array}{l}0 \\
0 \\
0 \\
0\end{array}\right|$ & $\begin{array}{l}2 \\
\dot{\sigma}_{\alpha} \\
\sigma^{\prime}\end{array}$ & $\begin{array}{l}8 \\
8 \\
-\end{array}$ & $\begin{array}{l}0 \\
\hat{a} \\
0 \\
0\end{array}$ & $\frac{\mathfrak{f}}{\stackrel{f}{0}}$ & $\frac{\stackrel{\overbrace{}}{\mathbb{N}}}{0^{\circ}}$ & $\begin{array}{l}\infty \\
\stackrel{n}{2} \\
0 \\
0\end{array}$ \\
\hline
\end{tabular}

.$\Xi$

등

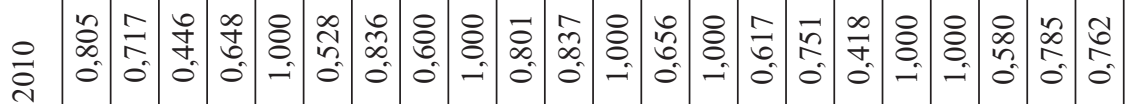

$\stackrel{\frac{\pi}{\pi}}{3}$

当

5

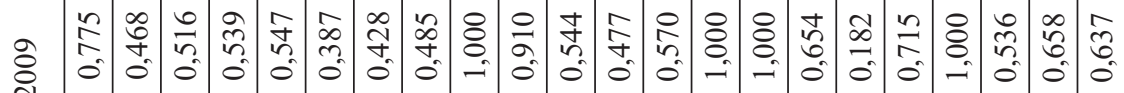

b

ฮี

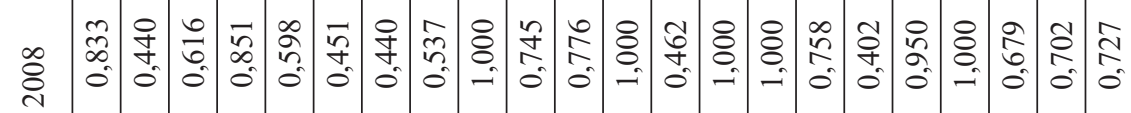

फ़े

깅

$\cdot$

(⿻)

E

$\ddot{\infty}$

$\ll$

$\frac{0}{0}$

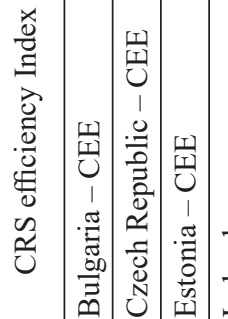

떧

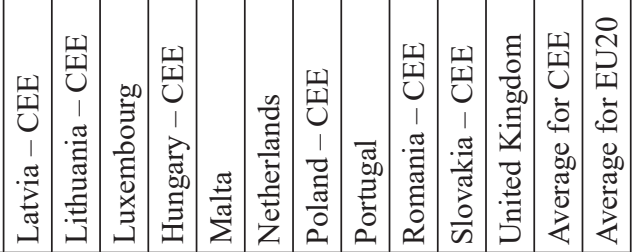

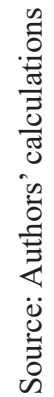


2
0
0
0
0
0
0
0
0
0
0
0
0
0

\begin{tabular}{|c|c|c|c|c|c|c|c|c|c|c|c|c|c|c|c|c|c|c|c|c|c|c|}
\hline $\begin{array}{l}\overrightarrow{0} \\
\overrightarrow{0} \\
\vec{G} \\
\vec{G}\end{array}$ & 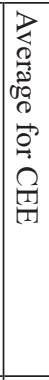 & 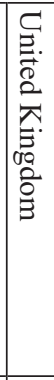 & 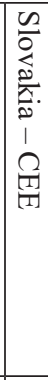 & 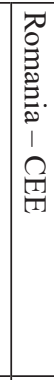 & 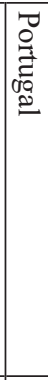 & 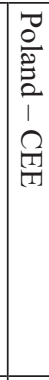 & 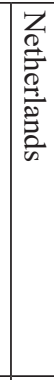 & $\mid \begin{array}{l}3 \\
\frac{2}{2} \\
2\end{array}$ & 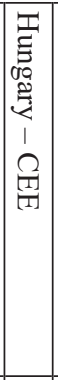 & 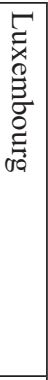 & 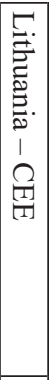 & 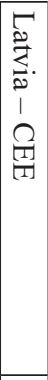 & 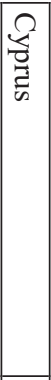 & $\overrightarrow{\overrightarrow{0}}$ & 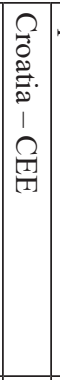 & 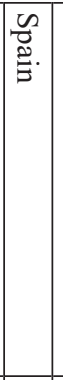 & \begin{tabular}{|l|} 
\\
\\
$\mathbb{8}$ \\
$\delta$ \\
$\delta$
\end{tabular} & 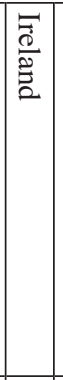 & 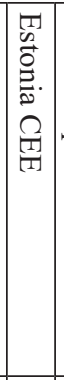 & 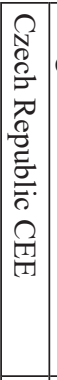 & 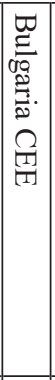 & 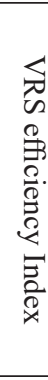 \\
\hline$\stackrel{0}{\circ} \underset{\infty}{0}$ & ○̃ & : & 5 & - & $\begin{array}{l}0 \\
\stackrel{0}{+} \\
\dot{f}\end{array}$ & $\begin{array}{l}\stackrel{0}{\sim} \\
\text { N }\end{array}$ & : & : & $\mid \begin{array}{l}0 \\
\ddot{\mathscr{g}} \\
\dot{8}\end{array}$ & : & 8 & 음 & $\stackrel{8}{8}$ & $\begin{array}{l}0 \\
\text { Un } \\
\text { 出 }\end{array}$ & 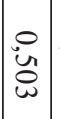 & $\mid \begin{array}{l}0 \\
0 \\
0 \\
\hat{\sigma}\end{array}$ & 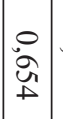 & : & 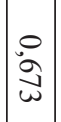 & $\mid \begin{array}{l}0 \\
ن \\
0 \\
0\end{array}$ & $\mid \begin{array}{l}0 \\
\dot{0} \\
\dot{\omega} \\
\mid\end{array}$ & $\underset{\infty}{\mathbb{8}}$ \\
\hline $\begin{array}{l}0 \\
\stackrel{0}{0}\end{array}$ & $\begin{array}{l}0 \\
\infty \\
\infty \\
0 \\
0\end{array}$ & $\stackrel{\circ}{\stackrel{0}{0}}$ & : & $\begin{array}{l}0 \\
0 \\
0 \\
0\end{array}$ & $\begin{array}{l}0 \\
\stackrel{\infty}{\infty} \\
\infty\end{array}$ & $\begin{array}{l}0 \\
\text { ᄋे }\end{array}$ & : & : & : & : & $\begin{array}{l}\circ \\
\stackrel{\circ}{N} \\
\end{array}$ & $\mid \begin{array}{l}0 \\
\dot{b} \\
\infty \\
+\infty\end{array}$ & : & $\stackrel{0}{0}$ & $\mid \begin{array}{l}0 \\
\dot{u} \\
\tilde{\omega} \\
\sigma\end{array}$ & $\left|\begin{array}{l}0 \\
\dot{\omega} \\
\delta \\
\delta\end{array}\right|$ & $\begin{array}{l}0 \\
\stackrel{0}{u} \\
0 \\
\omega\end{array}$ & $\begin{array}{l}0 \\
\infty \\
\infty \\
0 \\
u\end{array}$ & $\begin{array}{l}0 \\
\infty \\
0 \\
0\end{array}$ & : & $\mid \begin{array}{l}0 \\
0 \\
0 \\
0 \\
0\end{array}$ & 용 \\
\hline $\begin{array}{l}0 \\
\infty \\
\infty \\
\varrho\end{array}$ & $\begin{array}{l}0 \\
\text { No } \\
\text { Nu }\end{array}$ & 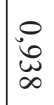 & : & 5 & 兑 & $\begin{array}{l}0 \\
\stackrel{0}{0} \\
\text { ర్ర }\end{array}$ & : & :5 & : & : & : & $\mid \begin{array}{l}0 \\
\infty \\
0 \\
0\end{array}$ & : & $\stackrel{0}{\circlearrowright}$ & $\mid \begin{array}{l}0 \\
\infty \\
\infty \\
\infty \\
\not\end{array}$ & $\begin{array}{l}\circ \\
\grave{\alpha} \\
\text { à }\end{array}$ & : & 명 & 잉 & : & $\mid \begin{array}{l}0 \\
\infty \\
N \\
N\end{array}$ & $\stackrel{N}{\stackrel{0}{\circ}}$ \\
\hline $\begin{array}{l}0 \\
\infty \\
\infty\end{array}$ & $\begin{array}{l}0 \\
\infty \\
\infty \\
\infty \\
\forall\end{array}$ & 范 & : & - & 品 & $\begin{array}{l}0 \\
\stackrel{0}{E}\end{array}$ & $\begin{array}{l}5 \\
8 \\
8\end{array}$ & 8 & : & : & $\begin{array}{l}\circ \\
\text { Nิ } \\
\text { م }\end{array}$ & $\begin{array}{l}\circ \\
\stackrel{0}{\infty} \\
\infty\end{array}$ & : & $\begin{array}{l}0 \\
\stackrel{2}{0} \\
\&\end{array}$ & 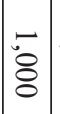 & 吕 & :5 & 명 & $\begin{array}{l}0 \\
\dot{w} \\
\vec{A}\end{array}$ & : & $\mid \begin{array}{l}0 \\
\dot{0} \\
\dot{\infty} \\
\infty\end{array}$ & $\stackrel{N}{\varrho}$ \\
\hline$\infty$ & $\stackrel{0}{0}$ & : & : & 5 & 虽 & $\begin{array}{l}\stackrel{\infty}{\infty} \\
\infty\end{array}$ & 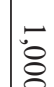 & 5 & $\begin{array}{l}\circ \\
\text { 崩 }\end{array}$ & 5 & : & $\mid \begin{array}{l}0 \\
0 \\
0 \\
0\end{array}$ & : & $\begin{array}{l}0 \\
\infty \\
\infty\end{array}$ & $\begin{array}{l}0 \\
\infty \\
\infty \\
\infty\end{array}$ & $\begin{array}{l}\circ \\
\dot{9}\end{array}$ & 它 & 5 & 怘 & 5 & {$\left[\begin{array}{l}0 \\
\infty \\
\infty\end{array} \mid\right.$} & $\frac{N}{N}$ \\
\hline
\end{tabular}

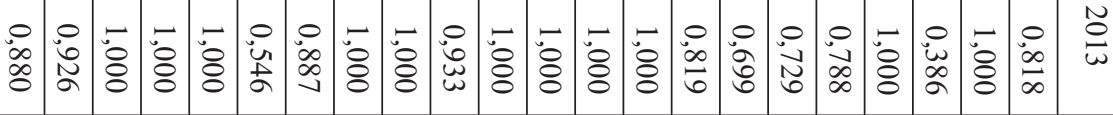

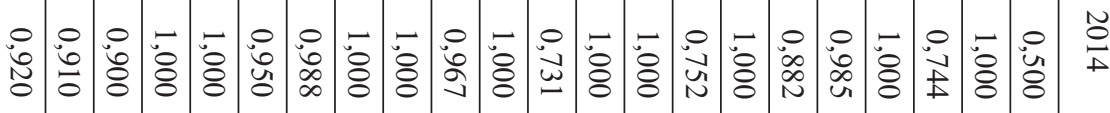

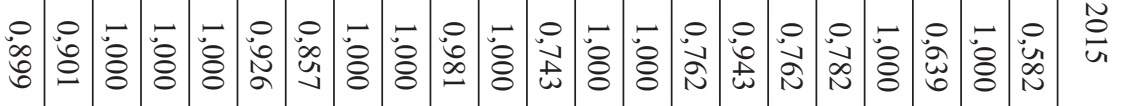

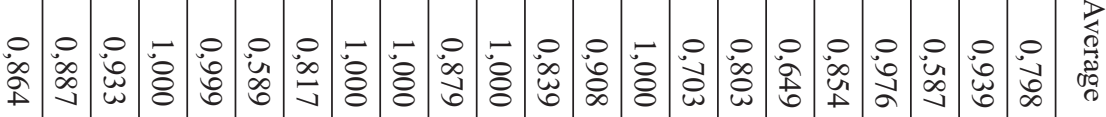

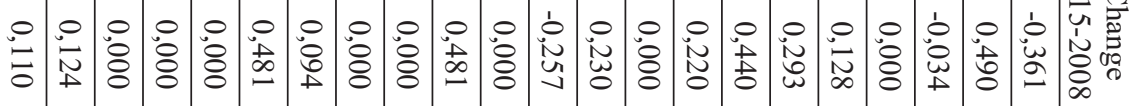


Pawel Dobrzanski • Innovation expenditures efficiency in Central and Eastern...

Zb. rad. Ekon. fak. Rij. • $2018 \cdot$ vol. $36 \cdot$ no. $2 \cdot 827-859$

Table A 10: Efficiency Ranking for EU20

\begin{tabular}{|l|c|c|c|c|}
\hline \multicolumn{1}{|c|}{ Country } & $\begin{array}{c}\text { Average CRS } \\
\text { Efficiency Index }\end{array}$ & $\begin{array}{c}\text { Average VRS } \\
\text { Efficiency Index }\end{array}$ & $\begin{array}{c}\text { Average } \\
\text { Efficiency Index }\end{array}$ & Rank \\
\hline Cyprus & 1,000 & 1,000 & 1,000 & 1 \\
\hline Malta & 0,982 & 1,000 & 0,991 & 2 \\
\hline Romania-CEE & 0,953 & 0,999 & 0,976 & 3 \\
\hline Slovakia-CEE & 0,942 & 1,000 & 0,971 & 4 \\
\hline Luxembourg & 0,907 & 1,000 & 0,953 & 5 \\
\hline Netherlands & 0,906 & 1,000 & 0,953 & 6 \\
\hline Latvia-CEE & 0,875 & 0,908 & 0,892 & 7 \\
\hline Ireland & 0,786 & 0,976 & 0,881 & 8 \\
\hline Greece & 0,782 & 0,854 & 0,818 & 9 \\
\hline United Kingdom & 0,686 & 0,933 & 0,810 & 10 \\
\hline Croatia-CEE & 0,733 & 0,803 & 0,768 & 11 \\
\hline Bulgaria-CEE & 0,736 & 0,798 & 0,767 & 12 \\
\hline Poland-CEE & 0,703 & 0,817 & 0,760 & 13 \\
\hline Lithuania-CEE & 0,679 & 0,839 & 0,759 & 14 \\
\hline Czech Republic-CEE & 0,566 & 0,939 & 0,752 & 15 \\
\hline Hungary - CEE & 0,622 & 0,879 & 0,750 & 16 \\
\hline Italy & 0,651 & 0,703 & 0,677 & 17 \\
\hline Spain & 0,566 & 0,649 & 0,607 & 18 \\
\hline Portugal & 0,485 & 0,589 & 0,537 & 19 \\
\hline Estonia-CEE & 0,482 & 0,587 & 0,534 & 20 \\
\hline Average for CEE & 0,729 & 0,887 & 0,808 & \\
\hline Average for EU20 & 0,752 & 0,864 & 0,808 & \\
\hline
\end{tabular}

Source: Authors' calculations 